\title{
On the calculation of energy release rate and mode mixity in delaminated laminated beams
}

\author{
Paolo S. Valvo ${ }^{a, *}$ \\ ${ }^{a}$ Department of Civil and Industrial Engineering, University of Pisa, Largo Lucio Lazzarino, I-56122 Pisa, Italy
}

\begin{abstract}
A method is presented for the analysis of laminated beams with general stacking sequences and arbitrarily located, through-the-width delaminations. First, the relative displacements and concentrated forces at the crack tip are determined based on classical lamination theory and Timoshenko beam kinematics. Next, new quantities, called crack-tip displacement rates, are defined as the relative displacements per unit increase in crack length. The previously computed quantities are then used to calculate the energy release rate and its mode I and II contributions via an adaptation of the virtual crack closure technique. Results for homogeneous and bimaterial delaminated beams are presented and compared to the predictions of other methods in the literature. Lastly, applications to some non-standard delamination test specimens are illustrated.
\end{abstract}

Keywords: Composites; Laminated beam; Delamination; Energy release rate; Mixed-mode fracture.

\section{Nomenclature}

\begin{tabular}{|c|c|}
\hline$a$ & (half) crack length \\
\hline$a, a_{1}, a_{2}$ & extensional compliances of laminate and sublaminates \\
\hline$A, A_{1}, A_{2}$ & extensional stiffnesses of laminate and sublaminates \\
\hline A, B & cross sections defining the crack-tip segment \\
\hline$b, b_{1}, b_{2}$ & bending-extension coupling compliances of laminate and sublaminates \\
\hline$b_{0}$ & unbalance parameter \\
\hline$B$ & laminate width \\
\hline$B, B_{1}, B_{2}$ & bending-extension coupling stiffnesses of laminate and sublaminates \\
\hline$C, c_{1}, c_{2}$ & shear compliances of laminate and sublaminates \\
\hline$C, C_{1}, C_{2}$ & initial crack-tip point positions \\
\hline$C, C_{1}, C_{2}$ & shear stiffnesses of laminate and sublaminates \\
\hline $\mathrm{C}$ & initial crack-tip cross section \\
\hline$d, d_{1}, d_{2}$ & bending compliances of laminate and sublaminates \\
\hline$D$ & crack-tip point after elementary crack growth \\
\hline$D, D_{1}, D_{2}$ & bending stiffnesses of laminate and sublaminates \\
\hline D & crack-tip cross section after elementary crack growth \\
\hline$E_{x}, E_{z}$ & Young's moduli in the $x$ - and $z$-directions \\
\hline$E_{x}^{(1)}, E_{x}^{(2)}, \ldots, E_{x}^{(n)}$ & Young's moduli of the layers of a laminated beam \\
\hline$f_{u M}, f_{u N}, f_{w Q}, f_{\phi M}, f_{\phi N}$ & flexibility coefficients \\
\hline$F_{1}, F_{2}, \ldots, F_{9}$ & integration constants for the elastic interface model \\
\hline$G$ & energy release rate \\
\hline$G_{c}$ & fracture toughness \\
\hline
\end{tabular}




\begin{tabular}{|c|c|}
\hline$G_{\mathrm{I}}, G_{\mathrm{II}}, G_{\mathrm{III}}$ & modal contributions to the energy release rate \\
\hline$G_{z x}$ & shear modulus in the $z x$-plane \\
\hline$G_{z x}^{(1)}, G_{z x}^{(2)}, \ldots, G_{z x}^{(n)}$ & shear moduli of the layers of a laminated beam \\
\hline$h, h_{1}, h_{2}$ & half thicknesses of laminate and sublaminates \\
\hline$H, H_{1}, H_{2}$ & thicknesses of laminate and sublaminates \\
\hline$i$ & layer index \\
\hline$k_{1}, k_{2}, k_{3}, k_{4}$ & coefficients by Schapery and Davidson [17] \\
\hline$k_{x}, k_{z}$ & elastic-interface constants \\
\hline$L$ & laminated beam length \\
\hline$M, M_{1}, M_{2}, M_{3}$ & bending moments in laminate and sublaminates \\
\hline$M_{1}^{C}, M_{2}^{C}$ & sublaminates bending moments in segment $S_{C}$ \\
\hline$M_{a}, M_{s}$ & antisymmetric and symmetric bending moments \\
\hline$M_{C}$ & crack-tip bending moment \\
\hline$M_{C}^{\mathrm{I}}, M_{C}^{\mathrm{II}}$ & mode I and mode II crack-tip bending moments \\
\hline $\bar{M}$ & applied bending moment on cantilever beam \\
\hline $\bar{M}_{1}, \bar{M}_{2}$ & applied bending moments in the DCB-UBM test \\
\hline$n$ & number of layers \\
\hline$N, N_{1}, N_{2}, N_{3}$ & axial forces in laminate and sublaminates \\
\hline$N_{1}^{C}, N_{2}^{C}$ & sublaminates axial forces in segment $S_{C}$ \\
\hline$N_{a}, N_{s}$ & antisymmetric and symmetric axial forces \\
\hline$N_{C}$ & crack-tip axial force \\
\hline$N_{C}^{\mathrm{I}}, N_{C}^{\mathrm{II}}$ & mode I and mode II crack-tip axial forces \\
\hline $\bar{N}$ & applied axial force on cantilever beam \\
\hline$O$ & origin of reference system \\
\hline$P$ & applied load in the ADCB test \\
\hline$P_{d}, P_{u}$ & downward and upward loads in the MMB test \\
\hline$Q, Q_{1}, Q_{2}, Q_{3}$ & shear forces in laminate and sublaminates \\
\hline$Q_{1}{ }^{C}, Q_{2}{ }^{C}$ & sublaminates shear forces in segment $S_{C}$ \\
\hline$Q_{a}, Q_{s}$ & antisymmetric and symmetric shear forces \\
\hline$Q_{C}$ & crack-tip transverse force \\
\hline$Q_{C}^{\mathrm{I}}, Q_{C}^{\mathrm{II}}$ & mode I and mode II crack-tip transverse forces \\
\hline $\bar{Q}$ & applied shear force on cantilever beam \\
\hline $\mathrm{S}, \mathrm{S}_{0}$ & crack-tip segment before and after elementary crack growth \\
\hline$S_{C}$ & crack-tip segment with crack-tip forces applied \\
\hline$t_{1}, t_{2}$ & thicknesses of layers in the MMB test with doublers \\
\hline$u, u_{1}, u_{2}$ & axial displacements of laminate and sublaminates \\
\hline$u_{C 1}, u_{C 2}$ & axial displacements of points $C_{1}$ and $C_{2}$ \\
\hline $\bar{u}$ & free-end axial displacement of cantilever beam \\
\hline$w, w_{1}, w_{2}$ & transverse displacements of laminate and sublaminates \\
\hline$w_{C 1}, w_{C 2}$ & transverse displacements of points $C_{1}$ and $C_{2}$ \\
\hline $\bar{w}$ & free-end transverse displacement of cantilever beam \\
\hline$x, y, z$ & coordinates \\
\hline$z_{0}, z_{1}, \ldots, z_{n}$ & ordinates of the top and bottom surfaces of layers \\
\hline$\alpha$ & laminate number \\
\hline$\alpha_{1}, \alpha_{2}, \alpha_{3}$ & coefficients of characteristic equation \\
\hline$\Delta a$ & crack length increment \\
\hline$\Delta u, \Delta w, \Delta \phi$ & crack-tip relative displacements \\
\hline$\varepsilon, \varepsilon_{1}, \varepsilon_{2}$ & mid-plane axial strains of laminate and sublaminates \\
\hline
\end{tabular}




\begin{tabular}{|c|c|}
\hline$\phi, \phi_{1}, \phi_{2}$ & cross sections' rotations of laminate and sublaminates \\
\hline$\phi_{C 1}, \phi_{C 2}$ & cross sections' rotations of points $C_{1}$ and $C_{2}$ \\
\hline $\bar{\phi}$ & free-end cross-section rotation of cantilever beam \\
\hline$\gamma, \gamma_{1}, \gamma_{2}$ & shear strains of laminate and sublaminates \\
\hline$\eta$ & sublaminates thickness ratio \\
\hline$\kappa, \kappa_{1}, \kappa_{2}$ & curvatures of laminate and sublaminates \\
\hline$\mu_{1}, \mu_{2}, \mu_{3}, \mu_{4}$ & parameters used to express the crack-tip forces \\
\hline$\mu_{x}, \mu_{z}$ & dimensionless elastic-interface constants \\
\hline$v_{1}, v_{2}, v_{3}, v_{4}$ & parameters used to express the crack-tip forces \\
\hline$v_{z x}$ & Poisson's ratio in the $z x$-plane \\
\hline$\rho_{u}, \rho_{w}, \rho_{\phi}$ & crack-tip displacement rates \\
\hline$\rho_{u}^{C}, \rho_{w}^{C}, \rho_{\phi}^{C}$ & crack-tip displacement rates in segment $S_{C}$ \\
\hline$\rho_{u}^{\mathrm{I}}, \rho_{w}^{\mathrm{I}}, \rho_{\phi}^{\mathrm{I}}$ & mode I crack-tip displacement rates \\
\hline$\rho_{u}^{\mathrm{II}}, \rho_{w}^{\mathrm{II}}, \rho_{\phi}^{\mathrm{II}}$ & mode II crack-tip displacement rates \\
\hline$\sigma_{0}$ & peak normal interfacial stress \\
\hline$\tau_{0}$ & peak tangential interfacial stress \\
\hline$\psi$ & mode-mixity angle \\
\hline$\Omega_{1}, \Omega_{2}, \Omega_{3}$ & roots of characteristic equation \\
\hline $\mathrm{ADCB}$ & asymmetric double cantilever beam \\
\hline AMMB & asymmetric mixed-mode bending \\
\hline DCB & double cantilever beam \\
\hline DCB-UBM & double cantilever beam loaded with uneven bending moments \\
\hline LEFM & linear elastic fracture mechanics \\
\hline MMB & mixed-mode bending \\
\hline VCCT & virtual crack closure technique \\
\hline
\end{tabular}

\section{Introduction}

Delamination is one of the most common and insidious failure modes for composite laminates. Interlaminar cracks propagating at the interface between two adjacent laminae may originate from different causes and lead to global failure of a laminate. A huge number of experimental, analytical, and numerical studies have been devoted to this problem during the last decades [1-5].

The analysis of delamination is commonly conducted in the context of fracture mechanics. In particular, based on linear elastic fracture mechanics (LEFM), the energy release rate, $G$, is the most used parameter to predict the initiation and growth of delamination cracks. On one hand, analytical and numerical models are developed to compute the expected values of $G$ in laboratory specimens or real structural components $[6,7]$. On the other hand, experimental techniques are used to assess the critical energy release rate, or interlaminar fracture toughness, $G_{\mathrm{c}}$, of specific materials [8]. The analysis is however complicated by the fact that delamination cracks usually propagate under a mix of the three basic fracture modes (I or opening, II or sliding, and III or tearing) and the fracture toughness depends on the mode 
mixity (a measure of the relative amounts of fracture modes). The energy release rate turns out to be the sum of three modal contributions, $G_{\mathrm{I}}, G_{\mathrm{II}}$, and $G_{\mathrm{III}}$, that the theoretical models should be able to predict. Besides, experimental testing should be carried out to determine the delamination toughness not only in pure fracture modes, but also under mixed-mode conditions. To this aim, several alternative theoretical models and experimental techniques have been developed. Their results, however, appear sometimes conflicting. Ducept et al. [9] conducted tests on symmetric and asymmetric delamination specimens. They analysed their experimental results based on the global method [10], local method [11], and virtual crack closure technique (VCCT) [12, 13], obtaining significant differences. Also, Harvey et al. [14, 15] observed relevant discrepancies between different fracture mode partitioning theories in the interpretation of experimental results.

In this paper, attention is focused on laminated beams containing delaminations under I/II mixed-mode conditions. This problem was analysed in a pioneering work by Pook [16], who used plate theory to calculate the energy release rate and the mode I and II stress intensity factors for several configurations of homogeneous and isotropic beams and plates with midplane delaminations. Williams [10] used beam theory to compute the energy release rate and the mode I and II contributions for homogeneous beams with delaminations located at arbitrary depth. Williams' global method assumes that fracture mode I is produced when opposite bending moments act on the two sublaminates into which the laminate is split; besides, mode II is assumed when the delaminating sublaminates have equal curvatures. Such assumptions, however, lead to incorrect results if the sublaminates have different thicknesses. To overcome this drawback, Schapery and Davidson [17] proposed a method based on classical laminated plate theory, where the mode mixity depends on a numerical parameter to be determined via finite element analysis. Independently, Nilsson and Storåkers [18] analysed layered plates and discussed several alternatives for the partitioning of fracture modes.

An elasticity theory approach was instead proposed by Suo and Hutchinson [11], who considered the problem of a semi-infinite crack between two homogeneous and isotropic elastic layers subjected to axial forces and bending moments. According to their local method, the energy release rate is computed based on beam theory, while the mode mixity is obtained by solving a plane elasticity problem. Suo [19] extended the local method to orthotropic materials by using the concept of orthotropy rescaling [20, 21]. Li et al. [22] and Andrews and Massabò [23] added the effects of shear forces for isotropic and orthotropic materials, respectively. In a recent paper, Li et al. [24] use classical beam theory and fitting of finite 
element results to reproduce Suo and Hutchinson's predictions for the mode mixity. They also present an interesting discussion on the relationship between the concentrated crack-tip forces of the beam-theory model and the singular stress distributions of the elasticity solution.

A different approach to the problem was proposed by Allix and Ladevèze [25], who modelled delamination by considering an elastic interface between the separating layers of a laminate. Subsequently, elastic-interface models were used many other authors [26-35]. Elastic-interface models can be regarded as particular cases of the more general class of cohesive-zone models [36-39]. Based on such models, the work of cohesive tractions (corresponding to the energy release rate of LEFM) is calculated based on the values of the interfacial stresses at the delamination front.

Most methods in the literature are limited to homogeneous or bimaterial beams. Only a few authors consider laminated beams. Amongst these, Carlsson et al. [40] and LundsgaardLarsen et al. [41] used classical lamination theory to analyse sandwich beams. Rask and Sørensen [42] proposed evaluating the $J$ integral for multidirectional laminated specimens from the measures of beam curvatures and bending moments. Wang and Harvey and coworkers proposed an orthogonal decomposition method to partition fracture modes in laminated beams [43-45].

The aim of this paper is to retrieve the spirit of Williams [10] and set up a method to calculate the energy release rate and mode mixity based solely on beam theory. Williams' assumptions on fracture mode partitioning are replaced by new assumptions, which hold also for laminates with delaminations not placed on the mid-plane. Furthermore, the proposed method applies not only to homogeneous and isotropic beams, but also to laminated beams with general stacking sequences, including those with bending-extension coupling.

The mechanical model underlying the present method is on purpose kept as simple as possible in order to obtain simple analytical expressions for all the quantities of interest. This is done at the risk of affecting the accuracy of results, but in the hope that the proposed approach could help to shed light on a subject, which still raises some controversy in the literature. For instance, it is assumed that the delaminating sublaminates are perfectly clamped at the crack-tip cross section. Indeed, it is well known that taking into account the so-called root rotations [46] could be crucial to get accurate predictions of experimental test results (in particular, in terms of the displacements and deformations of laboratory specimens). Moreover, the present method is based on Timoshenko beam kinematics [47], which assumes that plane cross sections remain plane and undeformed, but may rotate freely with respect to the beam centreline. Thus, the present model unavoidably disregards some strain 
contributions (for instance, those related to the Poisson effect), which would require an elasticity approach to be taken into account. As a consequence, it is expected that the results of the present method - in particular, for the mode mixity - may differ from those of more complex analytical and numerical methods in the literature. Nonetheless, the present method can serve as a benchmark for the validation of more complex methods in the limit case where classical beam theory assumptions are fulfilled (see, in what follows, the comparison with the elastic-interface model). Advantages of the proposed method compared to other ones include also the following:

(i) laminated beams with arbitrary stacking sequences can be analysed, including those with bending-extension coupling; instead, most methods in the literature are limited to homogeneous and isotropic beams;

(ii) analytical expressions are obtained for the energy release rate and its mode I and II contributions; there is no need to resort to finite element analyses, numerical calculations, or numerical parameters given in tabulated form;

(iii) shear forces and the effects of shear deformability are taken into account, which may be particularly relevant for composite laminates;

(iv) the method can be used for the design and data reduction of non-standard delamination toughness tests, i.e. tests on multidirectional laminated specimens with possibly asymmetric delaminations.

The structure of the paper is the following. In Section 2, a laminated beam is considered with a general stacking sequence and an arbitrarily located, through-the-width delamination. Attention is focused on a crack-tip segment, namely an infinitesimally short portion of the beam including one of the delamination crack tips. Based on classical lamination theory [48] and Timoshenko beam kinematics [47], the relative displacements occurring at the crack tip (after an elementary crack growth) and the concentrated forces acting at the crack tip (prior to the elementary crack growth) are determined. Furthermore, new quantities, called crack-tip displacement rates, are defined as the relative displacements per unit increase in crack length [49]. In Section 3, the crack-tip displacement rates are used to calculate the energy release rate and its modal contributions via an adaptation of the virtual crack closure technique $[13,50]$. Section 4 presents results for homogeneous and bimaterial delaminated beams. The present method is compared to the elastic-interface model [35] and the local method [11, 22]. Also, applications to some non-standard delamination test specimens with general stacking sequences are illustrated. Discussion of the obtained results follows in Section 5. 


\section{Static and kinematic analysis of a crack-tip segment}

\subsection{Delaminated laminated beam and crack-tip segment}

Let us consider a laminated beam of length $L$, thickness $H=2 h$, and width $B$, with an arbitrarily located, through-the-width delamination of length $2 a$ (Fig. 1). A Cartesian reference system Oxyz is fixed with the origin at the geometric centre of one of the end sections of the beam, the $x$-axis in the longitudinal direction, the $y$-and $z$-axes aligned with the width and height directions, respectively. It is assumed that geometry, material properties, restraints, and loads are such that the beam can be modelled as a planar beam in the $O z x$ plane. Within such assumptions, the beam can be made of layers of different materials and thicknesses and/or fibre-reinforced laminae with different orientations. Quite general stacking sequences are allowed by the present model, including those with bending-extension coupling, provided that the laminate and the sublaminates resulting from delamination are free from out-of-plane effects (e.g. torsion, out-of-plane shear, etc.). As an example, many symmetric and non-symmetric angle-ply and cross-ply laminates can be analysed.

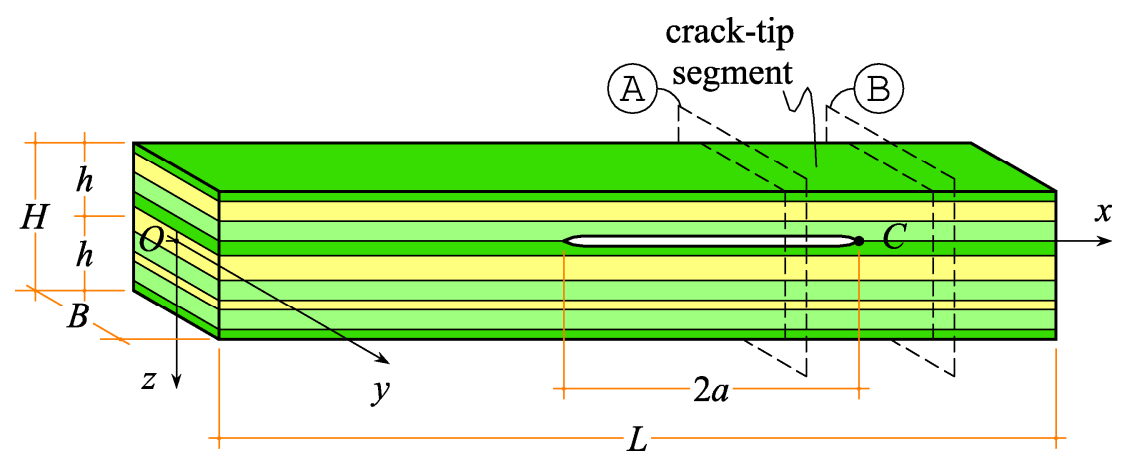

Fig. 1. Delaminated laminated beam and crack-tip segment.

Attention is now restricted to a crack-tip segment, defined as an infinitesimally short portion of the beam included between two cross sections, A and B, located immediately behind and ahead of the delamination crack tip, $C$ (Fig. 2). The crack-tip segment can be thought of as an assemblage of three short laminates, connected to each other at the crack-tip cross section, C (Fig. 2a): the upper and lower parts of the delaminated beam, denoted as sublaminates No. 1 and 2, respectively, and the undelaminated part, denoted as laminate No. 3. The sublaminate thicknesses are indicated with $H_{1}$ and $H_{2}$. Besides, in order to simplify some of the forthcoming expressions, the half thicknesses, $h_{1}=H_{1} / 2$ and $h_{2}=H_{2} / 2$, are introduced.

According to classical lamination theory [48], a laminated beam can be modelled as a homogeneous beam, provided that equivalent stiffnesses are defined (see also Appendix A). 
Hence, let $A_{\alpha}, B_{\alpha}, C_{\alpha}$, and $D_{\alpha}$ respectively denote the extensional stiffness, bendingextension coupling stiffness, shear stiffness, and bending stiffness per unit width of the three laminates (Fig. 2b). Here, the subscript $\alpha \in\{1,2,3\}$ refers to the laminate number.

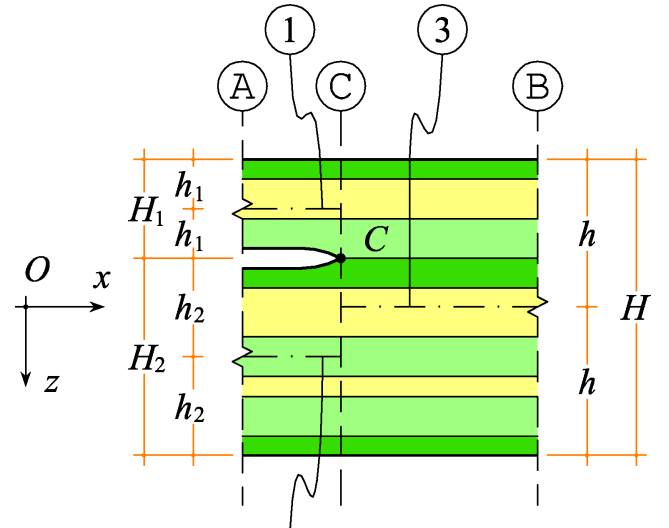

(a)

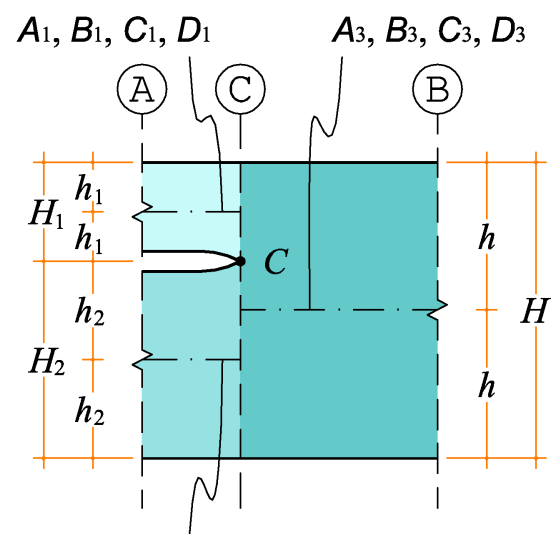

(b) $\quad A_{2}, B_{2}, C_{2}, D_{2}$

Fig. 2. Crack-tip segment: (a) laminated beam; (b) assemblage of equivalent homogeneous beams.

The internal forces are defined as the resultant forces acting on the laminate centrelines. Accordingly, the axial force, shear force, and bending moment are denoted with $N_{\alpha}, Q_{\alpha}$, and $M_{\alpha}$, respectively (Fig. 3). It is assumed that no external loads are applied on the crack-tip segment, which therefore will be in equilibrium under the action of the internal forces only. In the limit case of a crack-tip segment of vanishing length, the internal forces in laminate No. 3 turn out to be

$N_{3}=N_{1}+N_{2}, \quad Q_{3}=Q_{1}+Q_{2}, \quad$ and $\quad M_{3}=M_{1}+M_{2}-N_{1} h_{2}+N_{2} h_{1}$.

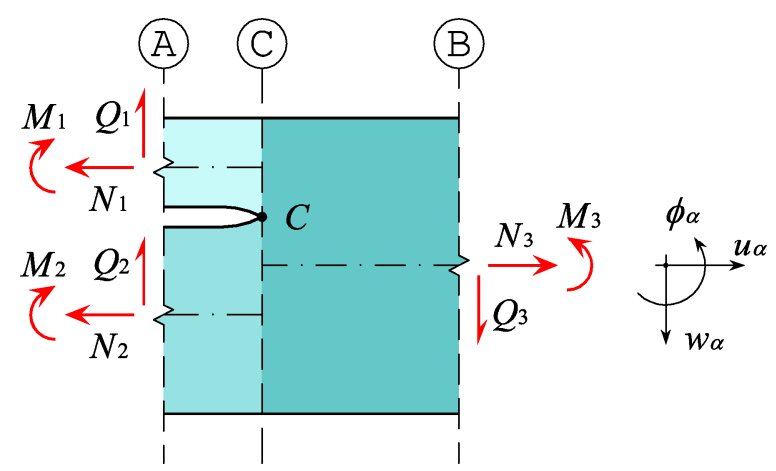

Fig. 3. Internal forces and generalised displacement convention.

Let $u_{\alpha}$ and $w_{\alpha}$ respectively denote the axial and transverse displacements of the laminate centreline, and $\phi_{\alpha}$ their cross-section rotation, positive if counter-clockwise (Fig. 3). In line with the Timoshenko beam theory [47], the axial strain, shear strain, and curvature are 
respectively defined as

$\varepsilon_{\alpha}=\frac{d u_{\alpha}}{d x}, \quad \gamma_{\alpha}=\frac{d w_{\alpha}}{d x}+\phi_{\alpha}$, and $\kappa_{\alpha}=\frac{d \phi_{\alpha}}{d x}$

According to classical lamination theory [48], the internal forces and strain measures can be related as follows:

$N_{\alpha}=B\left(A_{\alpha} \varepsilon_{\alpha}+B_{\alpha} \kappa_{\alpha}\right), \quad Q_{\alpha}=B C_{\alpha} \gamma_{\alpha}, \quad$ and $\quad M_{\alpha}=B\left(B_{\alpha} \varepsilon_{\alpha}+D_{\alpha} \kappa_{\alpha}\right)$,

or, by inversion of Eqs. (3),

$\varepsilon_{\alpha}=\frac{1}{B}\left(a_{\alpha} N_{\alpha}+b_{\alpha} M_{\alpha}\right), \quad \gamma_{\alpha}=\frac{1}{B} c_{\alpha} Q_{\alpha}, \quad$ and $\quad \kappa_{\alpha}=\frac{1}{B}\left(b_{\alpha} N_{\alpha}+d_{\alpha} M_{\alpha}\right)$,

where

$a_{\alpha}=\frac{D_{\alpha}}{A_{\alpha} D_{\alpha}-B_{\alpha}^{2}}, \quad b_{\alpha}=-\frac{B_{\alpha}}{A_{\alpha} D_{\alpha}-B_{\alpha}^{2}}, \quad c_{\alpha}=\frac{1}{C_{\alpha}}, \quad$ and $\quad d_{\alpha}=\frac{A_{\alpha}}{A_{\alpha} D_{\alpha}-B_{\alpha}^{2}}$

are the extensional compliance, bending-extension coupling compliance, shear compliance, and bending compliance, respectively. Note that if the sublaminates are uncoupled (which is the case for homogeneous and unidirectional laminates), $B_{\alpha}=0$ and $b_{\alpha}=0$, so that Eqs. (3)(5) can be conveniently simplified.

\subsection{Crack-tip relative displacements}

Let us now consider an elementary crack growth, whereby the delamination crack increases its length by a small amount, $\Delta a$, under constant external loads. The initial cracktip segment, S, where the crack tip is located at point $C$ (Fig. 4a), transforms into a new segment, $\mathrm{S}_{0}$, where the crack tip moves to a new position, $D$, while point $C$ splits into two points, $C_{1}$ and $C_{2}$, belonging to sublaminates No. 1 and 2, respectively (Fig. 4b). The cross section passing through point $D$ is denoted with $\mathrm{D}$. It is assumed that the internal forces acting on cross sections $\mathrm{A}$ and $\mathrm{B}$ do not change appreciably with the elementary crack growth, but points $C_{1}$ and $C_{2}$ generally undergo non-zero relative displacements (Fig. 5). The crack-tip relative displacements are defined as follows:

$\Delta u=u_{C 2}-u_{C 1}=\left(u_{2}-\phi_{2} h_{2}\right)-\left(u_{1}+\phi_{1} h_{1}\right)$,
$\Delta w=w_{C 2}-w_{C 1}=w_{2}-w_{1}, \quad$ and
$\Delta \phi=\phi_{C 2}-\phi_{C 1}=\phi_{2}-\phi_{1}$,

where $u_{C 1}$ and $u_{C 2}$ are the axial displacements of points $C_{1}$ and $C_{2}$, respectively, $w_{C 1}$ and 
$w_{C 2}$ are the corresponding transverse displacements, and $\phi_{C 1}$ and $\phi_{C 2}$ are the cross-section rotations. In particular, $\Delta u$ is the crack-tip sliding displacement, $\Delta w$ is the crack-tip opening displacement, and $\Delta \phi$ is the crack-tip relative rotation.

(S)

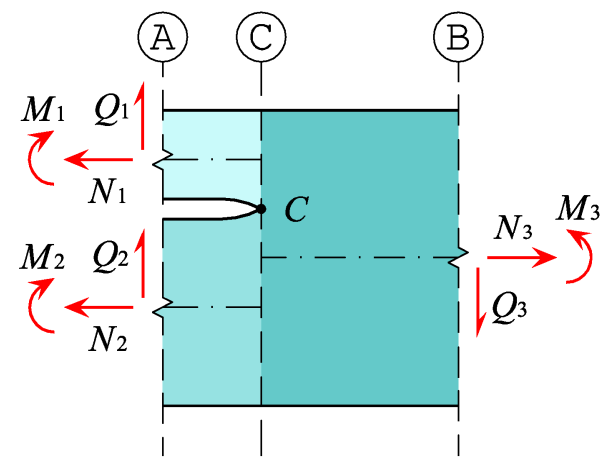

(a)
(S0)

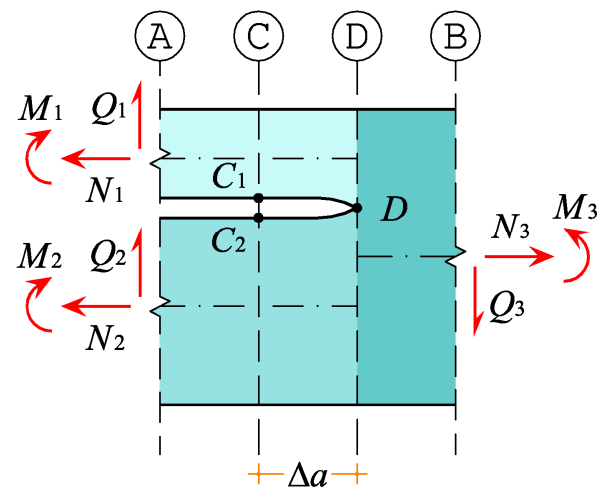

(b)

Fig. 4. Elementary crack growth: (a) initial crack-tip segment; (b) new crack-tip segment.

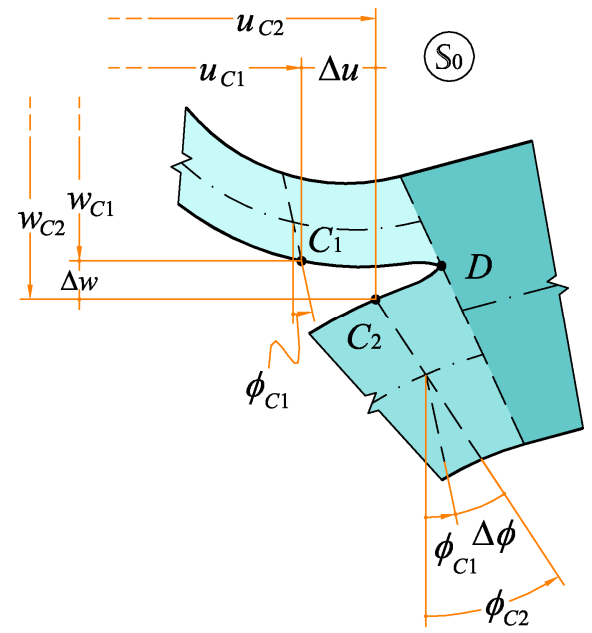

Fig. 5. Deformed crack-tip segment after the elementary crack growth.

The crack-tip relative displacements are here evaluated by assuming that the two delaminating sublaminates deform as cantilever beams of length $\Delta a$, clamped at cross section D and loaded by the internal forces, $N_{1}, Q_{1}, M_{1}$ and $N_{2}, Q_{2}, M_{2}$, at the cross sections passing through points $C_{1}$ and $C_{2}$. From the solution of the auxiliary problem of a laminated cantilever beam loaded at its end (see Appendix B), it is easily shown that 
$\Delta u \cong \frac{1}{B}\left[\left(a_{1}+b_{1} h_{1}\right) N_{1}-\left(a_{2}-b_{2} h_{2}\right) N_{2}+\left(b_{1}+d_{1} h_{1}\right) M_{1}-\left(b_{2}-d_{2} h_{2}\right) M_{2}\right] \Delta a$,

$\Delta w \cong \frac{1}{B}\left(c_{1} Q_{1}-c_{2} Q_{2}\right) \Delta a$, and

$\Delta \phi \cong \frac{1}{B}\left(b_{1} N_{1}-b_{2} N_{2}+d_{1} M_{1}-d_{2} M_{2}\right) \Delta a$,

where powers of $\Delta a$ of order higher than one have been neglected.

The clamped-end assumption for the sublaminates is a quite strong assumption, that will affect the results. Nevertheless, this assumption is made here for the sake of simplicity. A possible improvement to the model could be obtained by considering the root rotations, for instance, by introducing rotational springs at the sublaminate end sections [46].

\subsection{Crack-tip displacement rates}

The crack-tip displacement rates are defined as the crack-tip relative displacements per unit increase in crack length [49]:

$\rho_{u}=\lim _{\Delta a \rightarrow 0} \frac{\Delta u}{\Delta a}, \quad \rho_{w}=\lim _{\Delta a \rightarrow 0} \frac{\Delta w}{\Delta a}, \quad$ and $\quad \rho_{\phi}=\lim _{\Delta a \rightarrow 0} \frac{\Delta \phi}{\Delta a}$.

In particular, $\rho_{u}$ is the crack-tip sliding displacement rate, $\rho_{w}$ is the crack-tip opening displacement rate, and $\rho_{\phi}$ is the crack-tip relative rotation rate. By substituting Eqs. (7) into (8), the crack-tip displacement rates turn out to be

$\rho_{u}=\frac{1}{B}\left[\left(a_{1}+b_{1} h_{1}\right) N_{1}-\left(a_{2}-b_{2} h_{2}\right) N_{2}+\left(b_{1}+d_{1} h_{1}\right) M_{1}-\left(b_{2}-d_{2} h_{2}\right) M_{2}\right]$,

$\rho_{w}=\frac{1}{B}\left(c_{1} Q_{1}-c_{2} Q_{2}\right)$, and

$\rho_{\phi}=\frac{1}{B}\left(b_{1} N_{1}-b_{2} N_{2}+d_{1} M_{1}-d_{2} M_{2}\right)$.

By substituting Eqs. (3) into (9), and expressing the laminate stiffnesses as functions of the compliances through inversion of Eqs. (5), the following expressions are also obtained:

$\rho_{u}=\varepsilon_{1}-\varepsilon_{2}+\kappa_{1} h_{1}+\kappa_{2} h_{2}, \quad \rho_{w}=\gamma_{1}-\gamma_{2}, \quad$ and $\quad \rho_{\phi}=\kappa_{1}-\kappa_{2}$,

which show that the crack-tip displacement rates are a measure of the difference in strains at the crack tip between the two delaminating sublaminates. The crack-tip displacement rates describe the kinematics of an elementary crack growth. They will be used in the following to calculate the energy release rate and partition fracture modes. It is interesting to note that the first of Eqs. (10) - by recalling also Eqs. (8) - turns out to be very similar to the expression 
used by Carlsson et al. [40] to evaluate the tangential relative displacement of the crack faces of a double cantilever beam (DCB) sandwich specimen. Also, it is worth mentioning that similar quantities have been tacitly used by Schapery and Davidson [17].

\subsection{Crack-tip forces}

The initial crack-tip segment $S$ (prior to the elementary crack growth) can be obtained by superimposing the segments $S_{0}$ (after the elementary crack growth) and $S_{C}$, where suitable self-equilibrated pairs of axial forces, $N_{C}$, shear forces, $Q_{C}$, and bending moments, $M_{C}$, are applied at points $C_{1}$ and $C_{2}$ (Fig. 6). Such crack-tip forces can be regarded as the forces acting at the crack tip prior to crack propagation. These are different from the internal forces acting on the crack-tip segment prior to crack growth, which are applied on the sublaminate centrelines. The axial and bending components, $N_{C}$ and $M_{C}$, were introduced by Schapery and Davidson [17]. Harvey and Wang [44] considered also the shear component, $Q_{C}$. Li et al. [24] interpreted the crack-tip forces as the resultants of the singular stress fields at the crack tip of the plane elasticity solution.
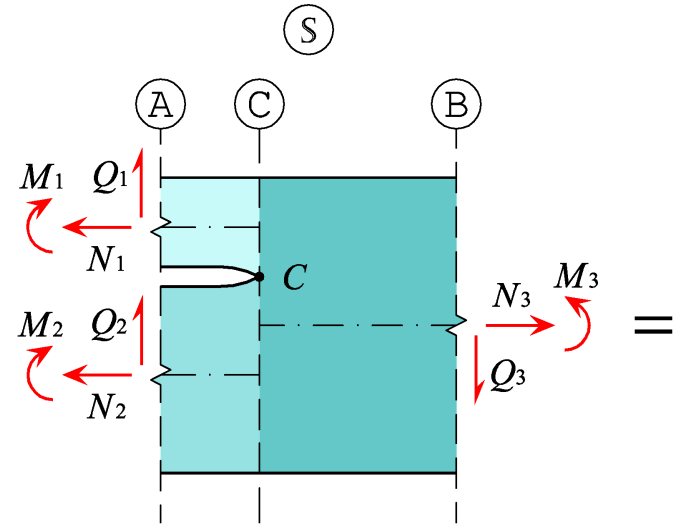

So)

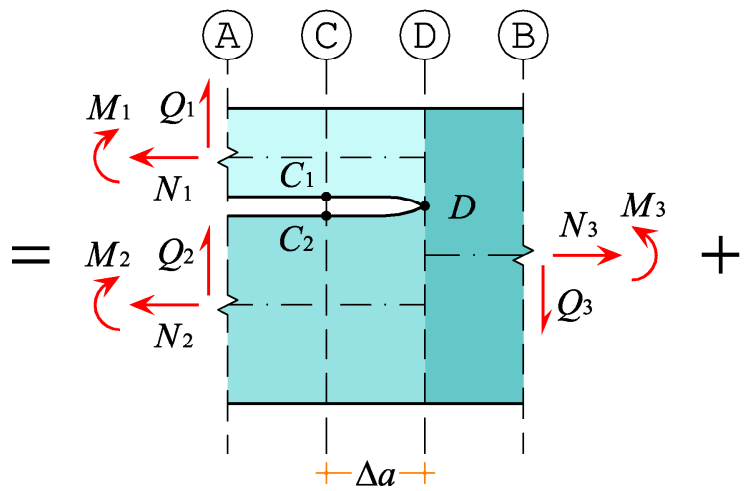

Sc)

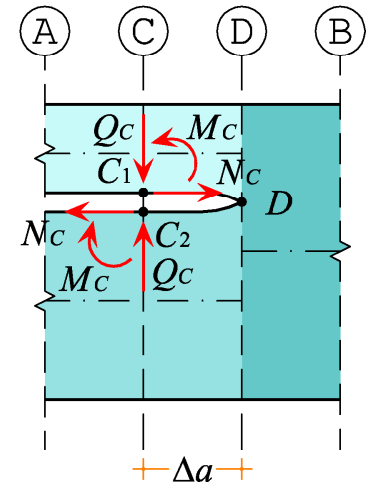

Fig. 6. The initial crack-tip segment $S$ as the superposition of segments $S_{0}$ and $S_{C}$.

The intensities of the crack-tip forces can be determined by observing that the crack-tip 
relative displacements resulting from the superposition of segments $S_{0}$ and $S_{C}$ should all be equal to zero. This is equivalent to impose the annihilation of the superimposed crack-tip displacement rates. Now, the internal forces in segment $S_{C}$ turn out to be

$N_{1}^{C}=-N_{C}, \quad Q_{1}^{C}=-Q_{C}, \quad$ and $\quad M_{1}^{C}=-M_{C}-N_{C} h_{1} ;$

$N_{2}^{C}=N_{C}, \quad Q_{2}^{C}=Q_{C}, \quad$ and $\quad M_{2}^{C}=M_{C}-N_{C} h_{2}$.

Hence, by substituting Eqs. (11) into (9), the crack-tip displacement rates in $S_{C}$,

$\rho_{u}^{C}=-f_{u N} N_{C}-f_{u M} M_{C}, \quad \rho_{w}^{C}=-f_{w Q} Q_{C}, \quad$ and $\quad \rho_{\phi}^{C}=-f_{\phi N} N_{C}-f_{\phi M} M_{C}$,

can be computed. In Eqs. (12) the flexibility coefficients,

$$
\begin{aligned}
& f_{u N}=\frac{1}{B}\left(a_{1}+a_{2}+2 b_{1} h_{1}-2 b_{2} h_{2}+d_{1} h_{1}^{2}+d_{2} h_{2}^{2}\right), \\
& f_{u M}=f_{\phi N}=\frac{1}{B}\left(b_{1}+b_{2}+d_{1} h_{1}-d_{2} h_{2}\right), \\
& f_{w Q}=\frac{1}{B}\left(c_{1}+c_{2}\right), \text { and } \\
& f_{\phi M}=\frac{1}{B}\left(d_{1}+d_{2}\right),
\end{aligned}
$$

have been introduced. The flexibility coefficients describe the deformability of the crack-tip segment. In passing, it is noted that similar quantities have been defined within a finite element framework in a revised formulation of the VCCT [51].

To annihilate the crack-tip relative displacements in $S$, it is necessary that

$$
\rho_{u}+\rho_{u}^{C}=0, \quad \rho_{w}+\rho_{w}^{C}=0, \quad \text { and } \quad \rho_{\phi}+\rho_{\phi}^{C}=0
$$

Hence, by substituting Eqs. (12) into (14), the crack-tip displacement rates,

$$
\rho_{u}=f_{u N} N_{C}+f_{u M} M_{C}, \quad \rho_{w}=f_{w Q} Q_{C}, \quad \text { and } \quad \rho_{\phi}=f_{\phi N} N_{C}+f_{\phi M} M_{C},
$$

are determined as functions of the crack-tip forces. It is noted that the coefficient $f_{u M}$ (equal to $f_{\phi N}$ by virtue of Betti's reciprocity theorem) introduces a coupling between $M_{C}$ and $\rho_{u}$ and, vice versa, between $N_{C}$ and $\rho_{\phi}$. This coupling vanishes for symmetrically delaminated beams with uncoupled sublaminates (for which $b_{1}=b_{2}=0$ and $d_{1} h_{1}=d_{2} h_{2}$ ) and, more generally, for delaminated laminated beams such that $b_{1}+d_{1} h_{1}=-b_{2}+d_{2} h_{2}$.

Lastly, by inversion of Eqs. (15), the crack-tip forces, 
$N_{C}=\frac{f_{\phi M} \rho_{u}-f_{u M} \rho_{\phi}}{f_{u N} f_{\phi M}-f_{u M} f_{\phi N}}, \quad Q_{C}=\frac{\rho_{w}}{f_{w Q}}, \quad$ and $\quad M_{C}=\frac{f_{u N} \rho_{\phi}-f_{\phi N} \rho_{u}}{f_{u N} f_{\phi M}-f_{u M} f_{\phi N}}$

are obtained as functions of crack-tip displacement rates. The crack-tip forces can also be expressed as functions of the internal forces in the sublaminates, as explained in Appendix C.

\section{Energy release rate and fracture mode partitioning}

\subsection{Energy release rate}

The energy release rate, $G$, is the decrease of potential energy of the system spent in the crack growth process, per unit area of new surface created. According to Irwin [52], the energy spent to produce an extension of the crack is equal to the work done to close the crack by the forces acting on the crack faces prior to crack extension. In analogy with the VCCT used in finite element analyses [12,13], the work of closure can be defined as the work done by the crack-tip forces on the corresponding relative displacements. Hence,

$G=\lim _{\Delta a \rightarrow 0} \frac{1}{2 B \Delta a}\left(N_{C} \Delta u+Q_{C} \Delta w+M_{C} \Delta \phi\right)$.

Schapery and Davidson [17] used an equation similar to Eq. (17), but without the shear term, $Q_{C} \Delta w$. This term is needed here because, according to the Timoshenko beam theory [47], there are three independent, generalised displacement components.

By recalling the definition of the crack-tip displacement rates, Eqs. (8), the energy release rate becomes

$G=\frac{1}{2 B}\left(N_{C} \rho_{u}+Q_{C} \rho_{w}+M_{C} \rho_{\phi}\right)$

Furthermore, by substituting Eqs. (15) into (18), the energy release rate can be expressed in terms of the crack-tip forces,

$G=\frac{1}{2 B}\left[f_{u N} N_{C}^{2}+\left(f_{\phi N}+f_{u M}\right) N_{C} M_{C}+f_{\phi M} M_{C}^{2}+f_{w Q} Q_{C}^{2}\right]$

or, by substituting Eqs. (16) into (18), in terms of the crack-tip displacement rates,

$G=\frac{1}{2 B}\left[\frac{f_{\phi M} \rho_{u}{ }^{2}-\left(f_{\phi N}+f_{u M}\right) \rho_{u} \rho_{\phi}+f_{u N} \rho_{\phi}{ }^{2}}{f_{u N} f_{\phi M}-f_{\phi N} f_{u M}}+\frac{\rho_{w}{ }^{2}}{f_{w Q}}\right]$.

Eqs. (18)-(20) offer three equivalent ways to compute the energy release rate for a given problem. In practice, once the internal forces at the cross sections behind and above of the 
crack tip are known, Eqs. (9) furnish the crack-tip displacement rates. Then, the crack-tip forces can be determined from Eqs. (16) and $G$ obtained via Eqs. (18) or (19). Alternatively, the energy release rate can be directly obtained from Eq. (20).

\subsection{Fracture mode partitioning}

Delamination cracks generally propagate under a mix of fracture modes. In I/II mixedmode problems, the energy release rate is the sum of two modal contributions,

$G=G_{\mathrm{I}}+G_{\mathrm{II}}$

respectively corresponding to fracture modes I (opening) and II (sliding). By inspection of Eqs. (18)-(20), it is not obvious how to identify the contributions of fracture modes I and II. On one hand, it is intuitive that the terms depending on $Q_{C}$ and $\rho_{w}$ should contribute only to $G_{\mathrm{I}}$ (incidentally, such terms are relevant only if shear deformability is taken into account); on the other hand, the terms depending on $N_{C}, M_{C}$ and $\rho_{u}, \rho_{\phi}$ are strongly tied one another and, hence, contribute to both fracture modes. It is also noted that this coupling depends on the flexibility coefficient $f_{u M}=f_{\phi N}$, which is generally non-zero in the case of asymmetric delamination cracks.

Here, the partitioning of fracture modes is based on the following simple kinematic assumption: pure mode I fracture occurs when the crack-tip sliding displacement, $\Delta u$, is equal to zero (or, equivalently, when the corresponding crack-tip displacement rate $\rho_{u}=0$ ).

The modal contributions to the energy release rate can then be computed in a way similar to that proposed elsewhere for the VCCT [50].

Let us imagine that the relative displacements between the crack faces are closed in an ideal two-step process. In the first ideal step, corresponding to the mode II contribution, suitable axial forces, $N_{C}^{\mathrm{II}}$, are applied at points $C_{1}$ and $C_{2}$, while neither shear forces, nor bending moments are applied at the same points (Fig. 7a). The intensity of $N_{C}^{\text {II }}$ should be such as to exactly close the gap in the $x$-direction, $\Delta u$, produced by the elementary crack growth. In practice, the first of Eqs. (15) can be used to determine $N_{C}^{\text {II }}$ as the axial force producing a crack-tip sliding displacement rate equal to $\rho_{u}$. To sum up, in the first ideal step the following crack-tip forces are applied:

$N_{C}^{\mathrm{II}}=\frac{\rho_{u}}{f_{u N}}, \quad Q_{C}^{\mathrm{II}}=0, \quad$ and $\quad M_{C}^{\mathrm{II}}=0$ 
Substitution of Eqs. (22) into (15) shows that the aforementioned crack-tip forces produce the following crack-tip displacement rates:

$\rho_{u}^{\mathrm{II}}=\rho_{u}, \quad \rho_{w}^{\mathrm{II}}=0, \quad$ and $\quad \rho_{\phi}^{\mathrm{II}}=\frac{f_{\phi N}}{f_{u N}} \rho_{u}$.

Eqs. (23) indicate that in the first ideal step, the gap between the crack faces in the $x$ direction is completely closed, the gap in the $z$-direction is left unchanged, while the relative rotation may be partly decreased or increased depending on the sign of the coupling flexibility coefficient, $f_{\phi N}$.

In the second ideal step, corresponding to mode I, the remainders of the crack-tip forces, $N_{C}^{\mathrm{I}}=N_{C}-N_{C}^{\mathrm{II}}, \quad Q_{C}^{\mathrm{I}}=Q_{C}, \quad$ and $\quad M_{C}^{\mathrm{I}}=M_{C}$,

are applied at points $C_{1}$ and $C_{2}$ (Fig. 7b). By substituting Eqs. (24) into (15) and recalling Eqs. (16) and (22), the crack-tip displacement rates produced by such crack-tip forces are determined as follows:

$\rho_{u}^{\mathrm{I}}=0, \quad \rho_{w}^{\mathrm{I}}=\rho_{w}, \quad$ and $\quad \rho_{\phi}^{\mathrm{I}}=\rho_{\phi}-\frac{f_{\phi N}}{f_{u N}} \rho_{u}$

(a)

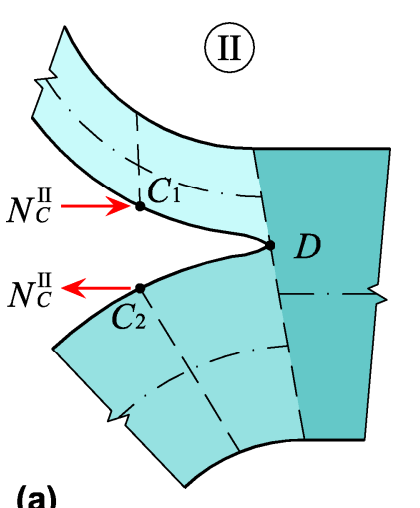

Fig. 7. Crack closure forces: (a) mode II; (b) mode I.

According to Eqs. (25), in the second ideal step, the gap in the $x$-direction (already closed in the first step) is left unchanged, while the gap in the $z$-direction is closed. Furthermore, from Eqs. (23) and (25) it follows that $\rho_{\phi}^{\mathrm{I}}+\rho_{\phi}^{\mathrm{II}}=\rho_{\phi}$, which means that the relative rotation between the crack faces vanishes as a result of the application of the crack-tip forces in the two ideal steps. Thus, the two ideal steps correspond to the complete closure of the crack.

The modal contributions to the energy release rate can now be determined by considering the work of closure done by the systems of crack-tip forces, Eqs. (22) and (24), on the 
corresponding crack-tip displacement rates, Eqs. (23) and (25). To this aim, it is worth noting that the two systems of crack-tip forces are energetically orthogonal, which means that their mutual work is zero $[50,51]$. Note also that $N_{C}^{\mathrm{II}}$ is in general distinct from $N_{C}$, but if $f_{u M}=f_{\phi N}=0$ then $\rho_{u}=f_{u N} N_{C}$ and $N_{C}^{\mathrm{II}}=N_{C}$. Eqs. (13) show that this happens when the unbalance parameter $b_{0}=b_{1}+b_{2}+d_{1} h_{1}-d_{2} h_{2}=0$. This condition includes, as special cases, homogeneous and unidirectional laminates with symmetrically located delaminations.

By substituting Eqs. (22)-(25) into (18), and recalling Eqs. (16), the energy release rate contributions corresponding to fracture modes I and II,

$$
G_{\mathrm{I}}=\frac{1}{2 B}\left[\frac{1}{f_{u N}} \frac{\left(f_{u N} \rho_{\phi}-f_{\phi N} \rho_{u}\right)^{2}}{f_{u N} f_{\phi M}-f_{u M} f_{\phi N}}+\frac{\rho_{w}^{2}}{f_{w Q}}\right] \text { and } G_{\mathrm{II}}=\frac{1}{2 B} \frac{\rho_{u}^{2}}{f_{u N}}
$$

are determined. By substituting Eqs. (15) into (26), the modal contributions can also be expressed as functions of the crack-tip forces:

$$
G_{\mathrm{I}}=\frac{1}{2 B}\left(\frac{f_{u N} f_{\phi M}-f_{u M} f_{\phi N}}{f_{u N}} M_{C}^{2}+f_{w Q} Q_{C}^{2}\right) \quad \text { and } \quad G_{\mathrm{II}}=\frac{1}{2 B} \frac{\left(f_{u N} N_{C}+f_{u M} M_{C}\right)^{2}}{f_{u N}} \text {. }
$$

Eqs. (26) and (27) show that both $G_{\mathrm{I}}$ and $G_{\mathrm{II}}$ are non-negative quantities, in line with their physical meaning [50,51]. Furthermore, the above equations reveal the conditions for having pure fracture modes: pure mode I $\left(G_{\mathrm{II}}=0\right)$ is obtained when $\rho_{u}=0$ (as per the initial assumption on fracture mode partitioning), while pure mode II $\left(G_{\mathrm{I}}=0\right)$ occurs when both $M_{C}=0$ and $Q_{C}=0$ (the latter condition is relevant only if shear deformability is taken into account). It is worth noting that the above conditions coincide with those obtained by Harvey and Wang [44] by using the Timoshenko beam theory [47]. Also, the above result is in line with the observation by Li et al. [24] that pure mode I cannot correspond to $N_{C}=0$, as this resultant force could vanish even if the distributed shear stresses at the crack tip are non-zero.

By recalling Eqs. (10), it is also possible to determine expressions for $G_{\mathrm{I}}$ and $G_{\mathrm{II}}$ as functions of the strain measures in the delaminating sublaminates,

$$
\begin{aligned}
& G_{\mathrm{I}}=\frac{1}{2 B}\left\{\frac{1}{f_{u N}} \frac{\left[f_{u N}\left(\kappa_{1}-\kappa_{2}\right)-f_{\phi N}\left(\varepsilon_{1}-\varepsilon_{2}+\kappa_{1} h_{1}+\kappa_{2} h_{2}\right)\right]^{2}}{f_{u N} f_{\phi M}-f_{u M} f_{\phi N}}+\frac{\left(\gamma_{1}-\gamma_{2}\right)^{2}}{f_{w Q}}\right\} \text { and } \\
& G_{\mathrm{II}}=\frac{1}{2 B} \frac{\left(\varepsilon_{1}-\varepsilon_{2}+\kappa_{1} h_{1}+\kappa_{2} h_{2}\right)^{2}}{f_{u N}} .
\end{aligned}
$$

Eqs. (28) shed some light on the discussion about the relationship between modal 
contributions and curvatures of the delaminating sublaminates [16-18, 42]. In particular, it is apparent that equal curvatures $\left(\kappa_{1}=\kappa_{2}\right)$ do not necessarily imply mode II fracture. On the other hand, opposite curvatures $\left(\kappa_{1}=-\kappa_{2}\right)$ correspond to mode I fracture, provided that the sublaminates have also equal axial strains $\left(\varepsilon_{1}=\varepsilon_{2}\right)$ and thicknesses $\left(h_{1}=h_{2}\right)$.

In I/II mixed-mode fracture problems, the mode mixity can be characterised by the phase angle of the complex stress-intensity factor [6]. Here, the mode-mixity angle is defined as $\psi= \pm \arctan \sqrt{\frac{G_{\mathrm{II}}}{G_{\mathrm{I}}}}$,

where the \pm sign has to be taken equal to the sign of $N_{C}^{\mathrm{II}}$ or $\rho_{u}$.

\section{Results}

\subsection{Homogeneous beams}

For homogeneous beams, the sublaminate stiffnesses are

$$
A_{\alpha}=E_{x} H_{\alpha}, \quad B_{\alpha}=0, \quad C_{\alpha}=\frac{5}{6} G_{z x} H_{\alpha}, \quad \text { and } \quad D_{\alpha}=\frac{1}{12} E_{x} H_{\alpha}^{3},
$$

where $E_{x}$ and $G_{z x}$ respectively are the longitudinal Young's modulus and shear modulus of the material. The subscript $\alpha=1$ denotes the upper sublaminate, $\alpha=2$ denotes the lower one. In what follows, also the elastic modulus in the transverse direction of the beam, $E_{z}$, will be used (for isotropic materials, $E_{x}=E_{z}$ ). By substituting Eqs. (30) into (5), (9), (13), and (20), the energy release rate,

$$
\begin{aligned}
G & =\frac{1}{2 B^{2} E_{x}} \frac{H_{1}^{3}+H_{2}^{3}}{H^{3}} \frac{H_{1} H_{2}}{H}\left(\frac{N_{1}}{H_{1}}-\frac{N_{2}}{H_{2}}\right)^{2}+\frac{3}{5 B^{2} G_{z x}} \frac{H_{1} H_{2}}{H}\left(\frac{Q_{1}}{H_{1}}-\frac{Q_{2}}{H_{2}}\right)^{2}+ \\
& +\frac{6}{B^{2} E_{x}}\left[\frac{M_{1}^{2}}{H_{1}^{3}}+\frac{M_{2}^{2}}{H_{2}^{3}}-\frac{\left(M_{1}+M_{2}\right)^{2}}{H^{3}}\right],
\end{aligned}
$$

is obtained as a function of the internal forces in the delaminating sublaminates. Likewise, from Eqs. (26), the mode I and II contributions, 


$$
\begin{aligned}
G_{\mathrm{I}} & =\frac{3}{8 B^{2} E_{x}}\left(H_{1}-H_{2}\right)^{2} \frac{H_{1} H_{2}}{H^{3}}\left(\frac{N_{1}}{H_{1}}-\frac{N_{2}}{H_{2}}\right)^{2}+\frac{3}{5 B^{2} G_{z x}} \frac{H_{1} H_{2}}{H}\left(\frac{Q_{1}}{H_{1}}-\frac{Q_{2}}{H_{2}}\right)^{2}+ \\
& +\frac{3}{2 B^{2} E_{x}} \frac{H_{1} H_{2}}{H^{3}}\left(\frac{3 H_{1}+H_{2}}{H_{1}^{2}} M_{1}-\frac{H_{1}+3 H_{2}}{H_{2}^{2}} M_{2}\right)^{2} \text { and } \\
G_{\mathrm{II}} & =\frac{1}{8 B^{2} E_{x}} \frac{H_{1} H_{2}}{H}\left(\frac{N_{1}}{H_{1}}-\frac{N_{2}}{H_{2}}\right)^{2}+\frac{9}{2 B^{2} E_{x}} \frac{H_{1} H_{2}}{H}\left(\frac{M_{1}}{H_{1}^{2}}+\frac{M_{2}}{H_{2}^{2}}\right)^{2},
\end{aligned}
$$

are determined as functions of the internal forces.

It can be shown that Eq. (31) is equivalent to the expressions by Williams [10], except for a term stemming from the moment balance of axial forces. However, the modal contributions given by Eqs. (32) are different from Williams' expressions because of the different underlying assumptions.

\subsubsection{Load cases}

In the following Sections, results for homogeneous beams will be presented and compared to the predictions of the elastic-interface model, where the two delaminating sublaminates are modelled as Timoshenko beams connected by an elastic interface [35] (see also Appendix D). Furthermore, the predictions of the local method $[11,22]$ will be shown for reference. In order to consider all possible load conditions, the general load system acting on the crack-tip segment is decomposed into the sum of three elementary load conditions, respectively corresponding to axial forces, shear forces, and bending moments applied to the delaminating sublaminates (Fig. 8). In turn, the elementary load conditions are decomposed into the sum of three symmetric load cases, where

$$
N_{s}=\frac{N_{1}+N_{2}}{2}, \quad Q_{s}=\frac{Q_{1}-Q_{2}}{2}, \quad \text { and } \quad M_{s}=\frac{M_{1}-M_{2}}{2},
$$

and three antisymmetric load cases, where

$$
N_{a}=\frac{N_{1}-N_{2}}{2}, \quad Q_{a}=\frac{Q_{1}+Q_{2}}{2}, \quad \text { and } \quad M_{a}=\frac{M_{1}+M_{2}}{2} \text {. }
$$



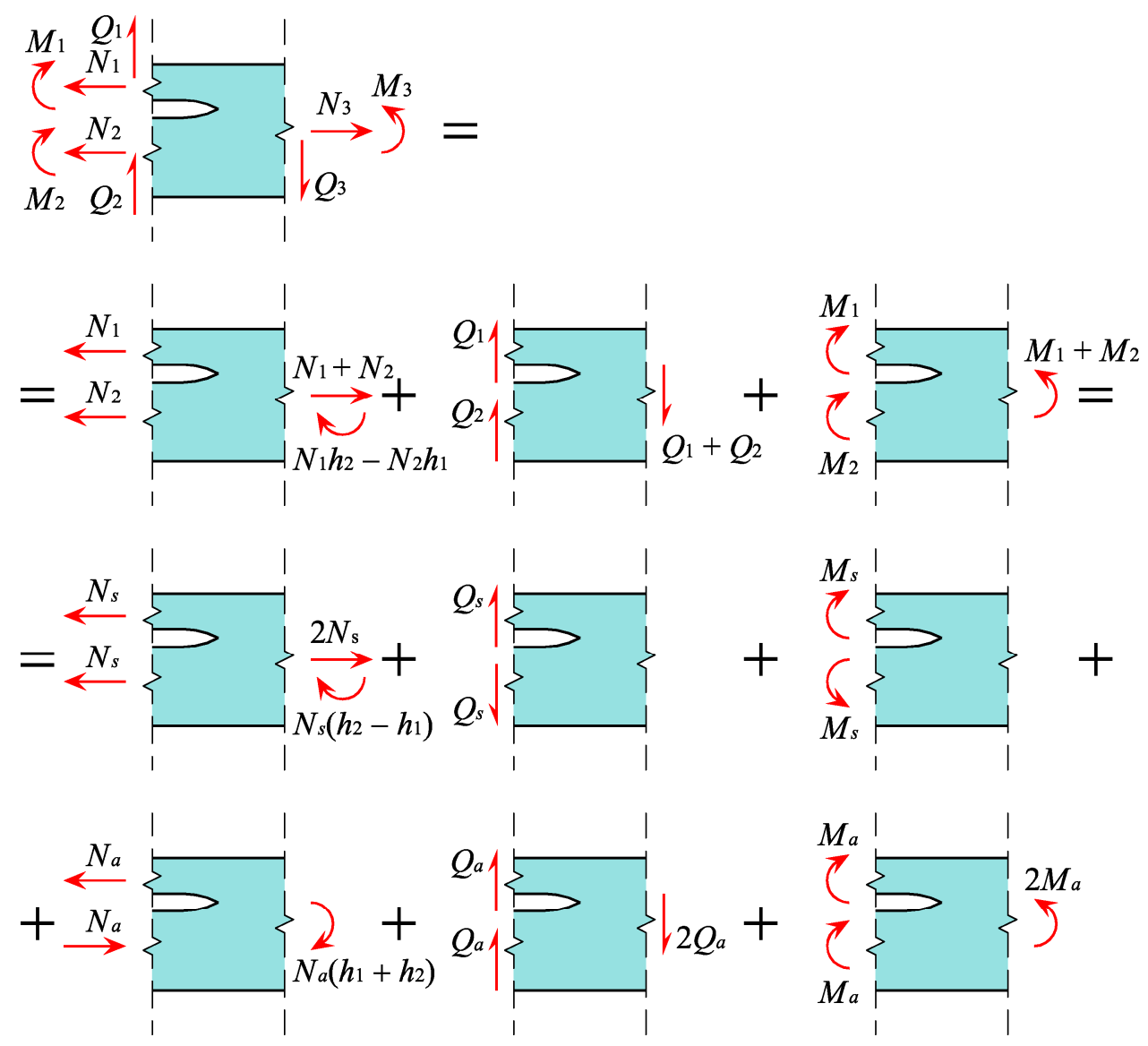

Fig. 8. Load decomposition for homogeneous beams.

It is worth noting that the symmetric and antisymmetric load cases correspond to fracture modes I and II, respectively, for laminates with symmetrically placed delaminations, but result in mixed-mode fracture in general. In what follows, the effects of axial forces, shear forces, and bending moments will be examined separately. For the sake of illustration, the Poisson's ratio is assumed as $v_{z x}=0.3$ and the shear modulus computed as $G_{z x}=E_{x} /\left[2\left(1+v_{z x}\right)\right]$.

\subsubsection{Effect of axial forces}

Figure 9 shows the energy release rate, $G$, produced by axial forces as a function of the sublaminate thickness ratio, $\eta=H_{1} / H_{2}$. As customary in the literature [22, 23], $G$ is nondimensionalised with respect to the applied load and the geometric and material parameters of the problem in order to make results independent of units of measurement. Without loss of generality, here and in the following, attention is restricted to the case $\eta \in[0,1]$. For both symmetric (Fig. 9a) and antisymmetric (Fig. 9b) axial forces, the energy release rate turns out to be a decreasing function of $\eta$ in the considered interval. The elastic-interface model [35] and local method [11] furnish for $G$ the same results of the present method. Note that for the 
symmetric load case, $G=0$ for $\eta=1$, which means that uniform traction does not produce fracture propagation in a symmetrically delaminated laminate.

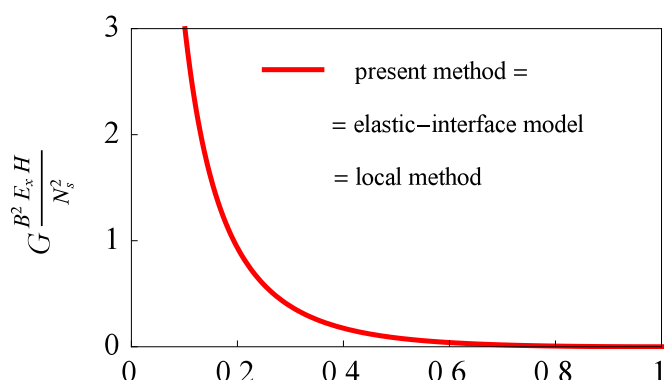

(a)

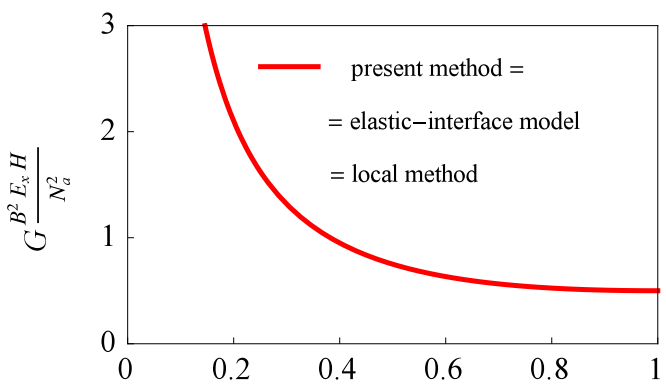

(b)

$\eta=\frac{H_{1}}{H_{2}}$

Fig. 9. Dimensionless energy release rate due to (a) symmetric and (b) antisymmetric axial forces.

Figure 10 shows the mode-mixity angle, $\psi$, produced by axial forces as a function of $\eta$. The plot is valid for both the symmetric and antisymmetric load cases. Actually, for axial forces, the mode mixity turns out to have the same dependency on the sublaminate thickness ratio in both cases. Results are presented for the present method (red, continuous curve), the elastic-interface model (orange, dashed curves), and the local method (blue, dotted curve). The results of the elastic-interface model depend on the values of the constants of the tangential and normal distributed springs, $k_{x}$ and $k_{z}$. The figure shows four curves corresponding to four values $(1,10,100$, and 1000) of the dimensionless elastic-interface constants, $\mu_{x}=k_{x} H / G_{z x}$ and $\mu_{z}=k_{z} H / E_{z}$. Note that as $\mu_{x}$ and $\mu_{z}$ increase, the predictions of the elastic-interface model tend towards those of the present method, to which they converge asymptotically for $\mu_{x}=\mu_{z} \rightarrow+\infty$ (rigid interface limit case). The predictions of the local method are approximately equal to those of the elastic-interface model for $\mu_{x}=\mu_{z}=10$ and $\eta \in[0.1,1]$. Major differences among the compared methods are observed for the smaller values of $\eta$. For $\eta \rightarrow 0$ (thin film limit case), the present method predicts mixed-mode fracture with $\psi=30^{\circ}$. This result is qualitatively consistent with the local method, which however predicts a different value of $\psi=52.1^{\circ}$. Instead, the elastic-interface model predicts pure mode II fracture $\left(\psi=90^{\circ}\right)$, regardless of the values of the interface constants. Interestingly, this latter result is consistent with the analysis of the peel test by De Lorenzis and Zavarise [53]. Lastly, it is noted that for $\eta=1$ (symmetric delamination), all the compared methods yield $\psi=90^{\circ}$, which means that if the fracture propagates $(G>0)$ then it does in mode II. 


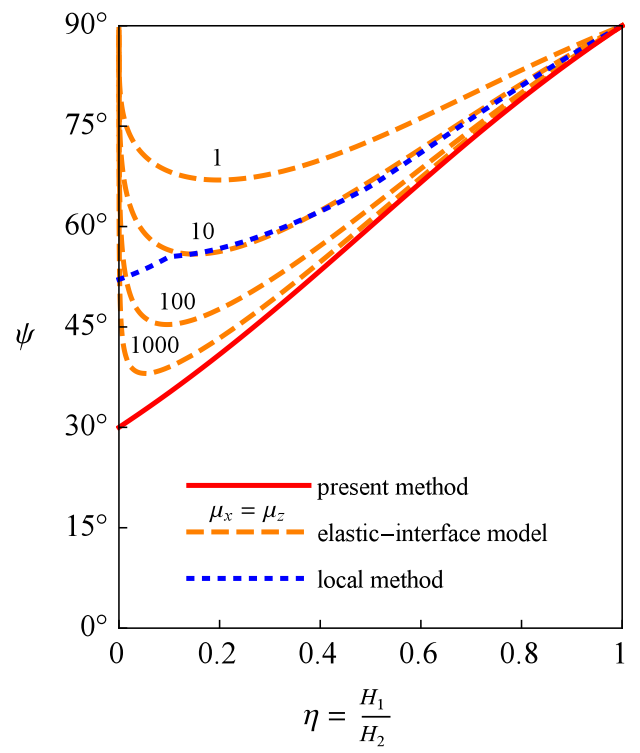

Fig. 10. Mode-mixity angle due to (symmetric and antisymmetric) axial forces.

\subsubsection{Effect of shear forces}

Figure 11 shows the energy release rate, $G$, produced by shear forces as a function of $\eta$. As for the axial force load case, $G$ is non-dimensionalised with respect to the parameters of the problem. All the compared methods predict the energy release rate to be a decreasing function of $\eta$ in the considered interval. For the elastic-interface model, four curves are shown, corresponding to four values $(1,10,100$, and 1000) of the dimensionless interface constants, $\mu_{x}$ and $\mu_{z}$. As the interface constants increase, the predictions of the elasticinterface model tend towards those of the present method, to which they converge asymptotically in the limit case of a rigid interface. Note that for the antisymmetric load case (Fig. 11b), the present method predicts $G=0$ for $\eta=1$ (uniform shear does not produce fracture propagation in a symmetrically delaminated laminate), but the compared methods predict $G>0$. This is likely because the other methods make different assumptions on the deformation of the crack-tip cross section. 

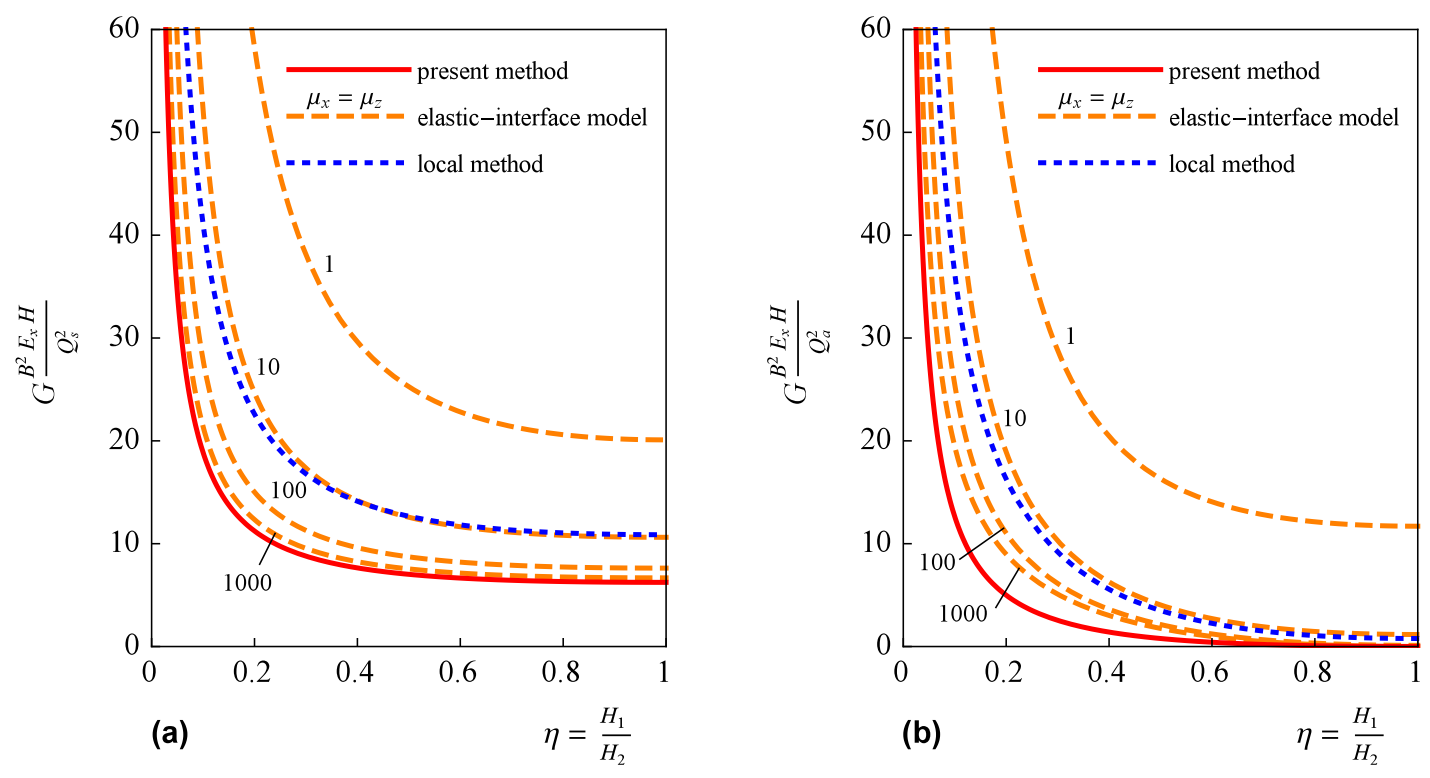

Fig. 11. Dimensionless energy release rate due to (a) symmetric and (b) antisymmetric shear forces.

Figure 12 shows the mode-mixity angle, $\psi$, produced by shear forces as a function $\eta$. The present method predicts $\psi=0^{\circ}$ (mode I fracture) for both the symmetric (Fig. 12a) and antisymmetric (Fig. 12b) load cases, for all values of $\eta$. This means that, according to the present method - based on the Timoshenko beam theory - shear forces do not contribute to $G_{\text {II }}$ [54]. Instead, both the elastic-interface model [35] and local method (in the version by Li et al. [22]) predict generally non-zero values for the mode-mixity angle. However, at least for the symmetric load case, the values of $\psi$ are very small (note the different axis scales in figures $12 \mathrm{a}$ and $12 \mathrm{~b}$ ). The predictions of the elastic-interface model (curves are plotted for $\mu_{x}$ and $\mu_{z}$ equal to $10,100,1000$, and $10^{4}$ ) tend towards those of the present method as $\mu_{x}=\mu_{z} \rightarrow+\infty$ (rigid interface limit case). The trends predicted by the local method appear qualitatively similar to those of the elastic-interface model, but quantitatively different. For $\eta=1$ (symmetric delamination), both the elastic-interface model and local method predict $\psi=0^{\circ}$ (mode I fracture) under symmetric shear loads and $\psi=90^{\circ}$ (mode II fracture) under antisymmetric shear loads. 

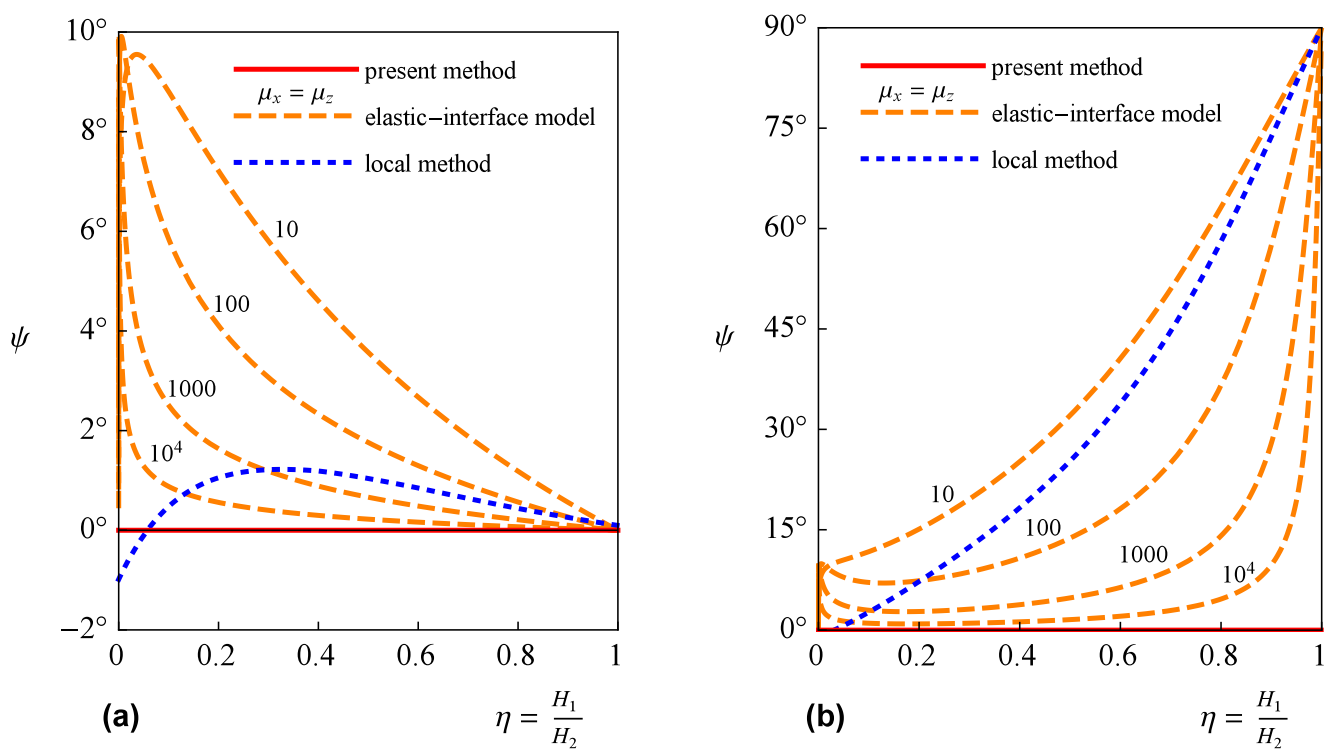

Fig. 12. Mode-mixity angle due to (a) symmetric and (b) antisymmetric shear forces.

\subsubsection{Effect of bending moments}

Figure 13 shows the energy release rate, $G$, produced by bending moments as a function of $\eta$. As for the previous load cases, $G$ is non-dimensionalised with respect to the parameters of the problem. For both symmetric (Fig. 13a) and antisymmetric (Fig. 13b) loads, the energy release rate is a decreasing function of $\eta$ in the considered interval. The elastic-interface model [35] and local method [11] furnish for $G$ the same results of the present method.

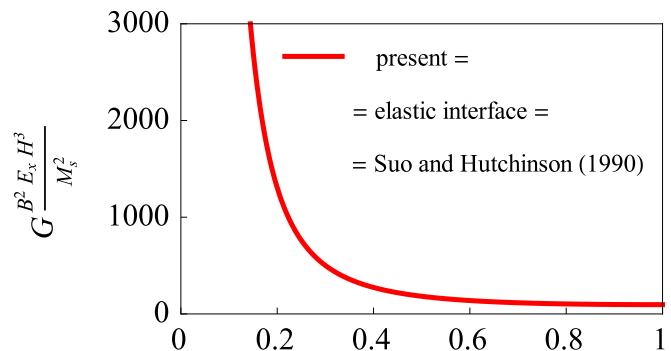

(a)

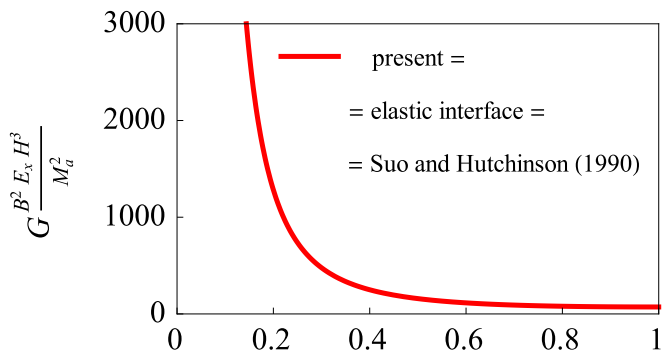

(b) $\eta=\frac{H_{1}}{H_{2}}$

Fig. 13. Dimensionless energy release rate due to (a) symmetric and (b) antisymmetric bending moments.

Figure 14 shows the mode-mixity angle, $\psi$, produced by symmetric and antisymmetric bending moments as a function of $\eta$. For the elastic-interface model, four values $(1,10,100$, and 1000) of the dimensionless interface constants, $\mu_{x}$ and $\mu_{z}$, are considered. Comments to figure 14 are similar to those applying to figure 10 (axial force load case). For $\eta \rightarrow 0$ (thin film limit case), the present method agrees qualitatively with the local method in predicting mixed-mode fracture, although they yield different values for $\psi\left(60^{\circ}\right.$ and $37.9^{\circ}$, respectively). 
The elastic-interface model predicts $\psi=0^{\circ}$ (mode I fracture), regardless of the values of the interface constants. This result is again consistent with the analysis of the peel test by De Lorenzis and Zavarise [53]. Lastly, it is noted that for $\eta=1$ (symmetric delamination), all the compared methods yield $\psi=0^{\circ}$ (mode I fracture) for symmetric loads and $\psi=90^{\circ}$ (mode II fracture) for antisymmetric loads.
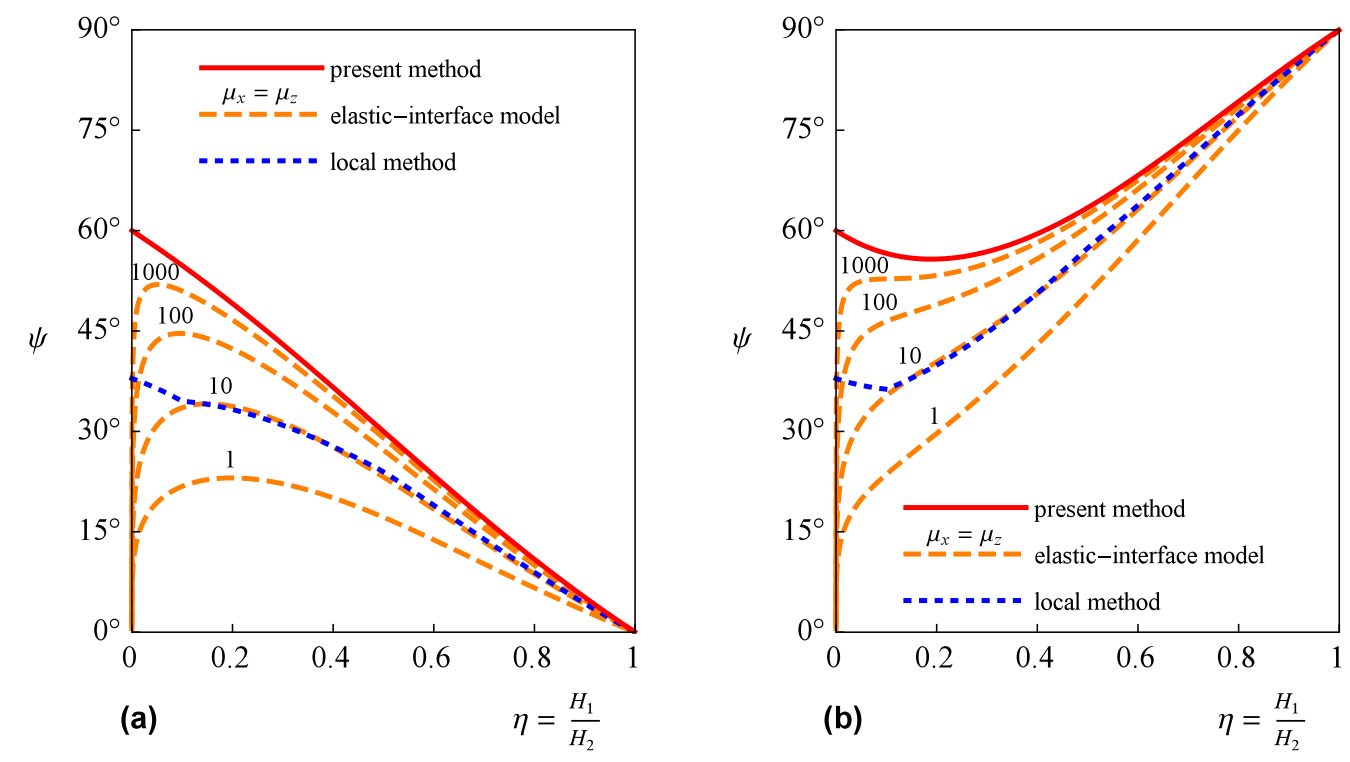

Fig. 14. Mode-mixity angle due to (a) symmetric and (b) antisymmetric bending moments.

\subsection{Bimaterial beams}

Bimaterial beams, namely laminated beams made of two different materials (Fig. 15), are now considered. For simplicity, each material is supposed to be homogeneous and isotropic. Furthermore, it is assumed that the two sublaminates have equal thicknesses $\left(H_{1}=H_{2}=h\right)$ in order to focus on the effects of the mismatch in the elastic moduli.

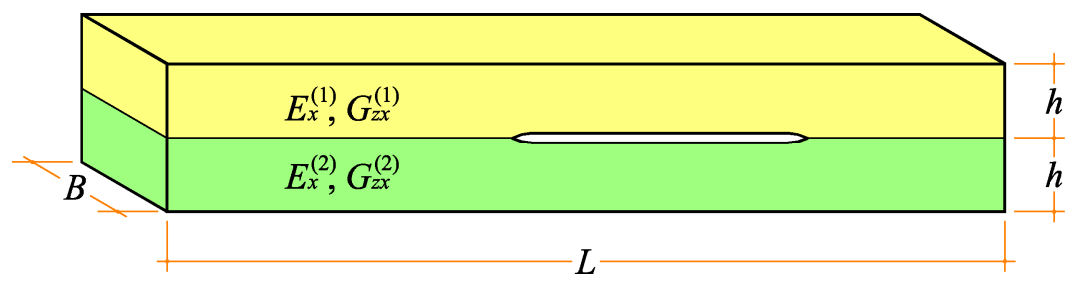

Fig. 15. Delaminated bimaterial beam.

The sublaminate stiffnesses are the following:

$$
\begin{aligned}
& A_{1}=E_{x}^{(1)} h, \quad B_{1}=0, \quad C_{1}=\frac{5}{6} G_{z x}^{(1)} h, \quad \text { and } \quad D_{1}=\frac{1}{12} E_{x}^{(1)} h^{3} ; \\
& A_{2}=E_{x}^{(2)} h, \quad B_{2}=0, \quad C_{2}=\frac{5}{6} G_{z x}^{(2)} h, \quad \text { and } \quad D_{2}=\frac{1}{12} E_{x}^{(2)} h^{3},
\end{aligned}
$$


where $E_{x}^{(1)}$ and $G_{z x}^{(1)}$ respectively are the Young's modulus and shear modulus of the upper sublaminate, $E_{x}^{(2)}$ and $G_{z x}^{(2)}$ are the corresponding quantities of the lower sublaminate. By substituting Eqs. (35) into (5), (9), (13), and (26), the mode I and II contributions to the energy release rate,

$$
\begin{aligned}
G_{\mathrm{I}} & =\frac{3}{8 B^{2} h^{3}} \frac{1}{E_{x}^{(1)}+E_{x}^{(2)}} \frac{1}{\frac{E_{x}^{(1)}}{E_{x}^{(2)}}+14+\frac{E_{x}^{(2)}}{E_{x}^{(1)}}} \\
& \cdot\left[\left(\frac{E_{x}^{(2)}}{E_{x}^{(1)}}-1\right) N_{1} h+\left(\frac{E_{x}^{(1)}}{E_{x}^{(2)}}-1\right) N_{2} h-2\left(\frac{E_{x}^{(2)}}{E_{x}^{(1)}}+7\right) M_{1}+2\left(\frac{E_{x}^{(1)}}{E_{x}^{(2)}}+7\right) M_{2}\right]^{2}+ \\
& +\frac{3}{5 B^{2} h} \frac{G_{z x}^{(1)} / G_{z x}^{(2)}}{G_{z x}^{(1)}+G_{z x}^{(2)}}\left(\frac{G_{z x}^{(2)}}{G_{z x}^{(1)}} Q_{1}-Q_{2}\right)^{2} \text { and } \\
G_{\mathrm{II}} & =\frac{1}{8 B^{2} h^{3}} \frac{E_{x}^{(1)} / E_{x}^{(2)}}{E_{x}^{(1)}+E_{x}^{(2)}}\left(\frac{E_{x}^{(2)}}{E_{x}^{(1)}} N_{1} h-N_{2} h+6 \frac{E_{x}^{(2)}}{E_{x}^{(1)}} M_{1}+6 M_{2}\right)^{2},
\end{aligned}
$$

are obtained as functions of the internal forces. The total energy release rate can be obtained by summing the two modal contributions given by Eqs. (36). It can be shown that the resulting expression for $G$ would be identical to the one by Suo and Hutchinson [11], for axial forces and bending moments, and to that by Andrews and Massabò [23], if also shear forces are considered (apart from a term stemming from root rotations, not considered here). Obviously, the modal contributions turn out to be different because of the different assumptions on fracture mode partitioning.

For the sake of illustration, four load cases are considered: antisymmetric axial forces, symmetric shear forces, symmetric and antisymmetric bending moments (Fig. 16). The Poisson's ratio is assumed as $v_{z x}=0.3$ and the shear modulus computed as $G_{z x}^{(\alpha)}=E_{x}^{(\alpha)} /\left[2\left(1+v_{z x}\right)\right]$, where $\alpha \in\{1,2\}$. 


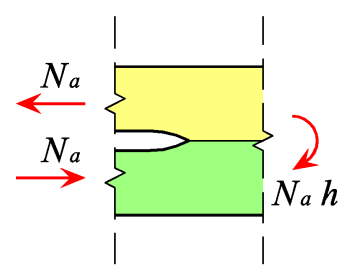

(a)

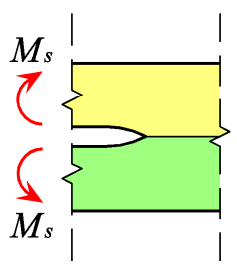

(c)

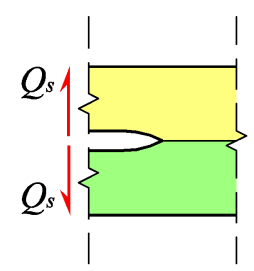

(b)

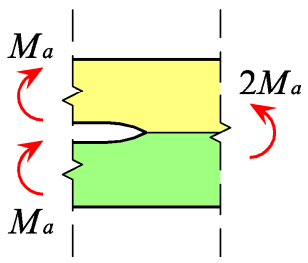

(d)

Fig. 16. Load cases for bimaterial beams: (a) antisymmetric axial forces; (b) symmetric shear forces; (c) symmetric bending moments; (d) antisymmetric bending moments.

Figure 17 shows the mode-mixity angle as a function of the ratio between the Young's moduli of the upper and lower sublaminates, $E_{x}^{(2)} / E_{x}^{(1)}$. The results of the present method are compared to those of the elastic-interface model [35] for three values of the dimensionless interface constants, $\mu_{x}=k_{x} H / G_{z x}^{(1)}$ and $\mu_{z}=k_{z} H / E_{x}^{(1)}$. It is noted that the predictions of the elastic-interface model tend towards those of the present method as $\mu_{x}=\mu_{z} \rightarrow+\infty$ (rigid interface limit case). For reference, also the predictions of the local method $[11,22]$ are plotted. The general trends are qualitatively similar, but differ quantitatively.

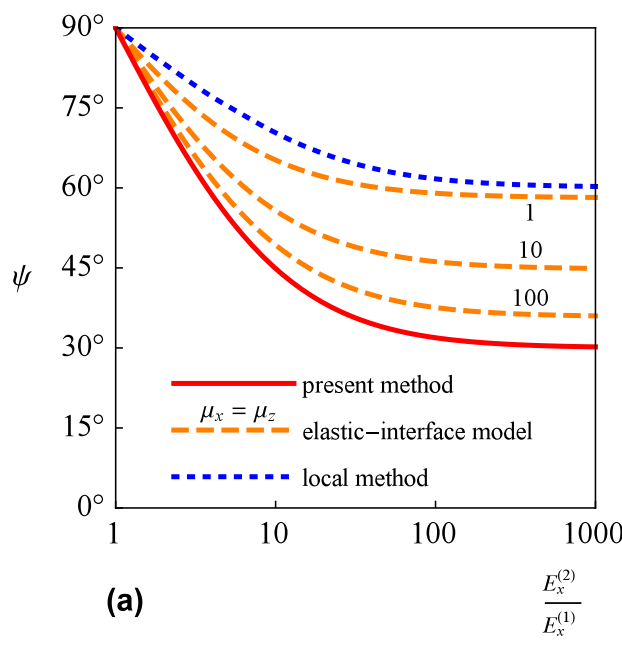

(a)

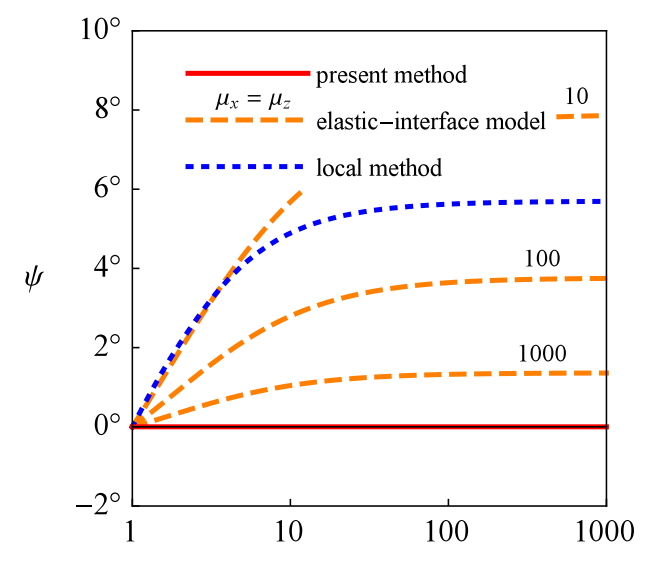

(b)

$\frac{E_{x}^{(2)}}{E_{x}^{(1)}}$ 

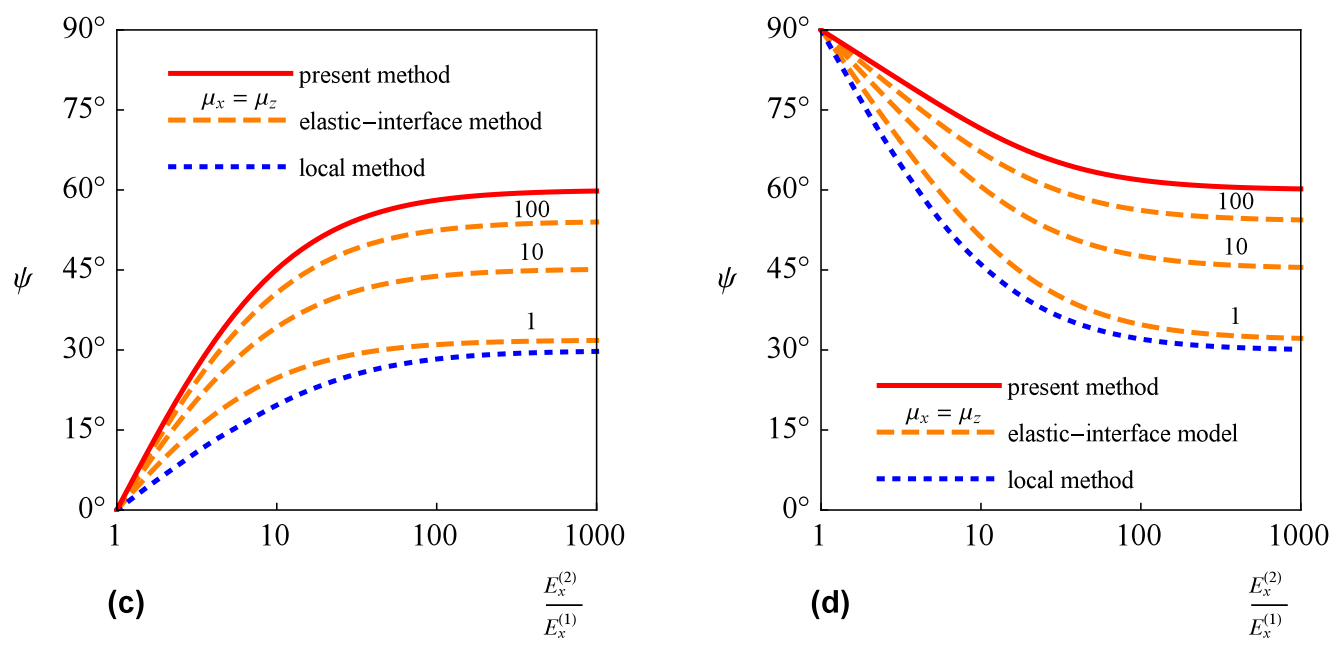

Fig. 17. Mode-mixity angle for bimaterial beams: (a) antisymmetric axial forces; (b) symmetric shear forces; (c) symmetric bending moments; (d) antisymmetric bending moments.

\subsection{Laminated beams}

For laminated beams with general stacking sequences, the energy release rate and mode mixity must be calculated by using the expressions of Section 3. The resulting general expressions are quite lengthy and will not be given here. However, as far as the total energy release rate is concerned, it can be shown that the present method yields exactly the same expression obtained by Schapery and Davidson [17] for the axial loads and bending moments contributions. Also, the expressions for the energy release rate obtained by Bao et al. [21] and Lundsgaard-Larsen et al. [41] for sandwich specimens can be retrieved.

By way of example, the method will be applied to some non-standard laboratory tests used to measure the delamination toughness of composite laminates:

- the asymmetric double cantilever beam (ADCB) test;

- the double cantilever beam loaded with uneven bending moments (DCB-UBM) test;

- the asymmetric mixed-mode bending (AMMB) test;

- the mixed-mode bending (MMB) test with doublers.

Lastly, a comparison with the method by Schapery and Davidson [17] will be made.

\subsubsection{Asymmetric double cantilever beam (ADCB) test}

In the asymmetric double cantilever beam test, a laminated specimen with an initial delamination of length $a$ is loaded by two opposite forces of equal intensity, $P$ (Fig. 18). The mode mixity originates from the geometric and/or material asymmetry of the loaded arms of the specimen $[31,55]$. 


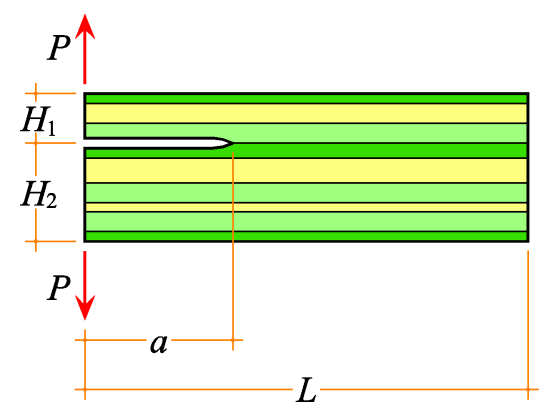

Fig. 18. Asymmetric double cantilever beam test.

The internal forces at the crack-tip cross section of an ADCB test specimen are

$N_{1}=0, \quad Q_{1}=P, \quad M_{1}=P a ;$

$N_{2}=0, \quad Q_{2}=-P, \quad M_{2}=-P a$.

By substituting Eqs. (37) into (9), the crack-tip displacement rates,

$\rho_{u}=\frac{P a}{B}\left(b_{1}+b_{2}+d_{1} h_{1}-d_{2} h_{2}\right), \quad \rho_{w}=\frac{P}{B}\left(c_{1}+c_{2}\right), \quad$ and $\quad \rho_{\phi}=\frac{P a}{B}\left(d_{1}+d_{2}\right)$,

are determined. Then, from Eqs. (13) and (16) the crack-tip forces,

$N_{C}=0, \quad Q_{C}=P, \quad$ and $\quad M_{C}=P a$,

are obtained. Lastly, from Eqs. (27) the mode I and II contributions to the energy release rate,

$G_{\mathrm{I}}=\frac{P^{2} a^{2}}{2 B^{2}}\left[d_{1}+d_{2}-\frac{\left(b_{1}+b_{2}+d_{1} h_{1}-d_{2} h_{2}\right)^{2}}{a_{1}+a_{2}+2 b_{1} h_{1}-2 b_{2} h_{2}+d_{1} h_{1}^{2}+d_{2} h_{2}^{2}}\right]+\frac{P^{2}}{2 B^{2}}\left(c_{1}+c_{2}\right)$ and

$G_{\mathrm{II}}=\frac{P^{2} a^{2}}{2 B^{2}} \frac{\left(b_{1}+b_{2}+d_{1} h_{1}-d_{2} h_{2}\right)^{2}}{a_{1}+a_{2}+2 b_{1} h_{1}-2 b_{2} h_{2}+d_{1} h_{1}^{2}+d_{2} h_{2}^{2}}$,

are calculated. The sum of the two modal contributions yields the total energy release rate,

$G=\frac{P^{2}}{2 B^{2}}\left[c_{1}+c_{2}+a^{2}\left(d_{1}+d_{2}\right)\right]$

It can be verified that, if the delaminating sublaminates are uncoupled $\left(b_{1}=b_{2}=0\right)$ and shear deformability is neglected ( $c_{1}=c_{2}=0$ ), then Eqs. (40) yield the same results computed by the elastic-interface model [31] in the limit case of a rigid interface $\left(\mu_{x}=\mu_{z} \rightarrow+\infty\right)$.

4.3.2. Double cantilever beam loaded with uneven bending moments (DCB-UBM) test

Sørensen et al. [56] have proposed a modified DCB test, where the specimen is loaded by bending moments, $\bar{M}_{1}$ and $\bar{M}_{2}$ (Fig. 19). Loading by moments instead of forces has several advantages: (i) the whole range of mode mixities (from pure mode I to mode II) can be tested 
by varying the load ratio, $\bar{M}_{1} / \bar{M}_{2}$; (ii) delamination growth is stable during the test for all mode mixities; (iii) the test results are independent of the delamination length (which can be difficult to measure in some circumstances); (iv) shear deformability does not influence the test results. For symmetric specimens, the symmetric and antisymmetric parts of the applied loads respectively correspond to fracture modes I and II. If the specimen is not symmetric about the delamination plane, then an additional source of mode mixity is given by the geometric and/or material asymmetry of the loaded arms. For the sake of simplicity, a symmetrically delaminated specimen is considered here $\left(a_{1}=a_{2}, b_{1}=-b_{2}, c_{1}=c_{2}, d_{1}=d_{2}\right.$, and $\left.h_{1}=h_{2}\right)$.

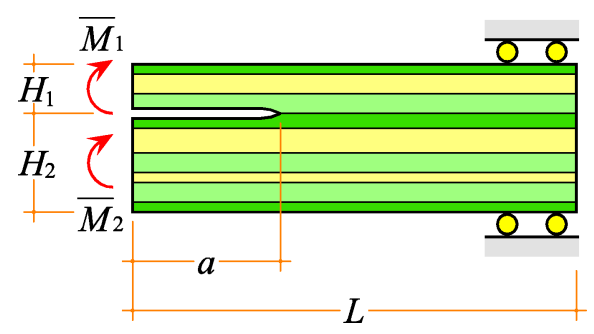

Fig. 19. Double cantilever beam loaded with uneven bending moments test.

The internal forces at the crack-tip cross section of a DCB-UBM test specimen are

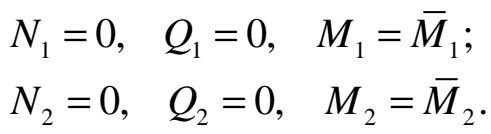

By substituting Eqs. (42) into (9), the crack-tip displacement rates,

$$
\rho_{u}=\frac{1}{B}\left(b_{1}+d_{1} h_{1}\right)\left(M_{1}+M_{2}\right), \quad \rho_{w}=0, \quad \text { and } \quad \rho_{\phi}=\frac{1}{B} d_{1}\left(M_{1}-M_{2}\right),
$$

are determined. Then, from Eqs. (13) and (16) the crack-tip forces,

$$
N_{C}=\frac{1}{2} \frac{b_{1}+d_{1} h_{1}}{a_{1}+2 b_{1} h_{1}+d_{1} h_{1}^{2}}\left(M_{1}+M_{2}\right), \quad Q_{C}=0, \quad \text { and } \quad M_{C}=\frac{1}{2}\left(M_{1}-M_{2}\right) \text {, }
$$

are obtained. Lastly, from Eqs. (27) the modal contributions to the energy release rate,

$$
G_{\mathrm{I}}=\frac{d_{1}}{4 B^{2}}\left(M_{1}-M_{2}\right)^{2} \text { and } G_{\mathrm{II}}=\frac{1}{4 B^{2}} \frac{\left(b_{1}+d_{1} h_{1}\right)^{2}}{a_{1}+2 b_{1} h_{1}+d_{1} h_{1}^{2}}\left(M_{1}+M_{2}\right)^{2} \text {, }
$$

are calculated.

\subsubsection{Asymmetric mixed-mode bending (AMMB) test}

In the mixed-mode bending (MMB) test, a laminated specimen with an initial delamination 
of length $a$ is loaded simultaneously by an upward load, $P_{u}$, and a downward load, $P_{d}$ (Fig. 20). By suitably changing the relative intensities of the applied loads, a range of mode mixities can be tested [57]. The test is standardised for unidirectional laminated specimens with symmetric delaminations by ASTM [58], but has been used also for multidirectional and asymmetric specimens [9], bimaterial specimens [59, 60], multidirectional laminates $[32,33$, 61], sandwich plates [62], and asymmetric adhesively bonded joints [63, 64]. In all the aforementioned cases, where there is geometric and/or material asymmetry about the delamination plane, the partitioning of fracture modes does not correspond to the decomposition of the applied loads into the symmetric and antisymmetric parts, as it does for symmetrically delaminated specimens. Unfortunately, this point appears not to have been fully appreciated in the literature with a few exceptions $[32,33,61]$. The present method furnishes a way to determine the mode I and II contributions to the energy release rate for asymmetric MMB test specimens within the context of beam theory.

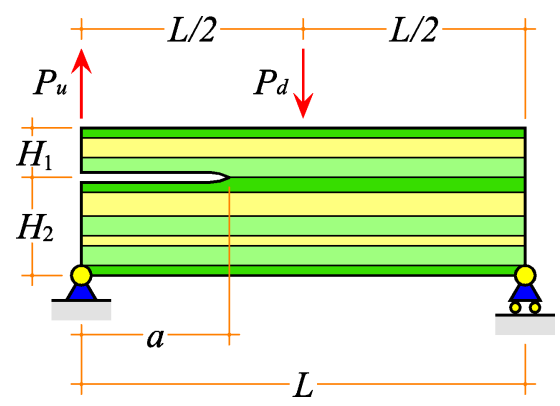

Fig. 20. Asymmetric mixed-mode bending test.

The internal forces at the crack-tip cross section of an AMMB test specimen are $N_{1}=0, \quad Q_{1}=P_{u}, \quad M_{1}=P_{u} a$ $N_{2}=0, \quad Q_{2}=\frac{1}{2} P_{d}-P_{u}, \quad M_{2}=\left(\frac{1}{2} P_{d}-P_{u}\right) a$.

By substituting Eqs. (46) into (9), the crack-tip displacement rates,

$\rho_{u}=\frac{P_{u} a}{B}\left(b_{1}+b_{2}+d_{1} h_{1}-d_{2} h_{2}\right)-\frac{P_{d} a}{2 B}\left(b_{2}-d_{2} h_{2}\right), \quad \rho_{w}=\frac{P_{u}}{B}\left(c_{1}+c_{2}\right)-\frac{P_{d}}{2 B} c_{2}$, and $\rho_{\phi}=\frac{P_{u} a}{B}\left(d_{1}+d_{2}\right)-\frac{P_{d} a}{2 B} d_{2}$,

are obtained. Such quantities can then be used to calculate the energy release rate and its modal contributions via Eqs. (20) and (26), respectively. However, the resulting general expressions are quite lengthy and will not be given here. 
4.3.4. Mixed-mode bending (MMB) test with doublers

Reeder et al. [65] have proposed to reinforce mixed-mode bending test specimens (of thickness $2 t_{2}$ ) experiencing premature bending failure by adding 'doublers', i.e. externally bonded sheets of stiffer material (of thickness $t_{1}$ ). The result is an MMB specimen, where the sublaminates are bimaterial beams with bending-extension coupling (Fig. 21).

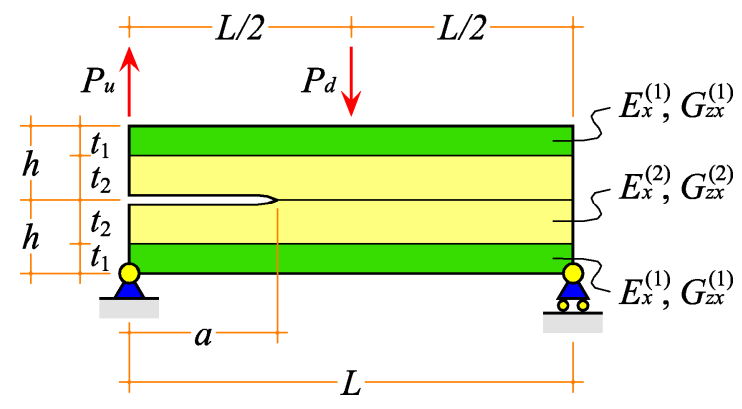

Fig. 21. Mixed-mode bending with doublers test.

The sublaminate equivalent stiffnesses turn out to be

$$
\begin{aligned}
& A_{1}=A_{2}=E_{x}^{(1)} t_{1}+E_{x}^{(2)} t_{2}, \quad B_{1}=-B_{2}=-\frac{1}{2}\left(E_{x}^{(1)}-E_{x}^{(2)}\right) t_{1} t_{2} \\
& C_{1}=C_{2}=\frac{5}{6}\left(G_{z x}^{(1)} t_{1}+G_{z x}^{(2)} t_{2}\right), \quad \text { and } \quad D_{1}=D_{2}=\frac{1}{12}\left[E_{x}^{(1)} t_{1}\left(t_{1}^{2}+3 t_{2}^{2}\right)-E_{x}^{(2)} t_{2}\left(t_{2}^{2}+3 t_{1}^{2}\right)\right]
\end{aligned}
$$

while the compliances can be calculated via Eqs. (5). By observing that the specimen is symmetric about the delamination plane $\left(a_{1}=a_{2}, b_{1}=-b_{2}, c_{1}=c_{2}, d_{1}=d_{2}\right.$, and $\left.h_{1}=h_{2}\right)$, the crack-tip displacement rates, Eqs. (47), can be simplified as follows:

$$
\rho_{u}=\frac{P_{d} a}{2 B}\left(b_{1}+d_{1} h_{1}\right), \quad \rho_{w}=\frac{4 P_{u}-P_{d}}{2 B} C_{1}, \quad \text { and } \quad \rho_{\phi}=\frac{\left(4 P_{u}-P_{d}\right) a}{2 B} d_{1} \text {. }
$$

By substituting Eqs. (49) into (26), and recalling Eqs. (13), the mode I and II contributions to the energy release rate,

$$
G_{\mathrm{I}}=\frac{\left(4 P_{u}-P_{d}\right)^{2}}{16 B^{2}}\left(c_{1}+a^{2} d_{1}\right) \text { and } G_{\mathrm{II}}=\frac{P_{d}^{2} a^{2}}{16 B^{2}} \frac{\left(b_{1}+d_{1} h_{1}\right)^{2}}{a_{1}+2 b_{1} h_{1}+d_{1} h_{1}^{2}}
$$

are obtained.

\subsubsection{Comparison with the method by Schapery and Davidson}

For general laminated beams, Schapery and Davidson [17] expressed the mode I and II contributions to the energy release rate as follows: 
$G_{\mathrm{I}}=\frac{1}{2 B}\left(k_{1} N_{C}+k_{2} M_{C}\right)^{2} \quad$ and $\quad G_{\mathrm{II}}=\frac{1}{2 B}\left(k_{3} N_{C}+k_{4} M_{C}\right)^{2}$

where $k_{1}, k_{2}, k_{3}$, and $k_{4}$ are real coefficients, which depend on the stacking sequence, but not on the load condition. The authors showed that $k_{2}, k_{3}$, and $k_{4}$ can be expressed as functions of $k_{1}$. The latter coefficient can be determined numerically by using the finite element method to solve the particular problem of a delaminated beam loaded with the following internal forces (Fig. 22):

$N_{1} \neq 0, \quad Q_{1}=0, \quad M_{1}=N_{1} h_{1}$

$N_{2}=-N_{1}, \quad Q_{2}=0, \quad M_{2}=N_{1} h_{2}$.

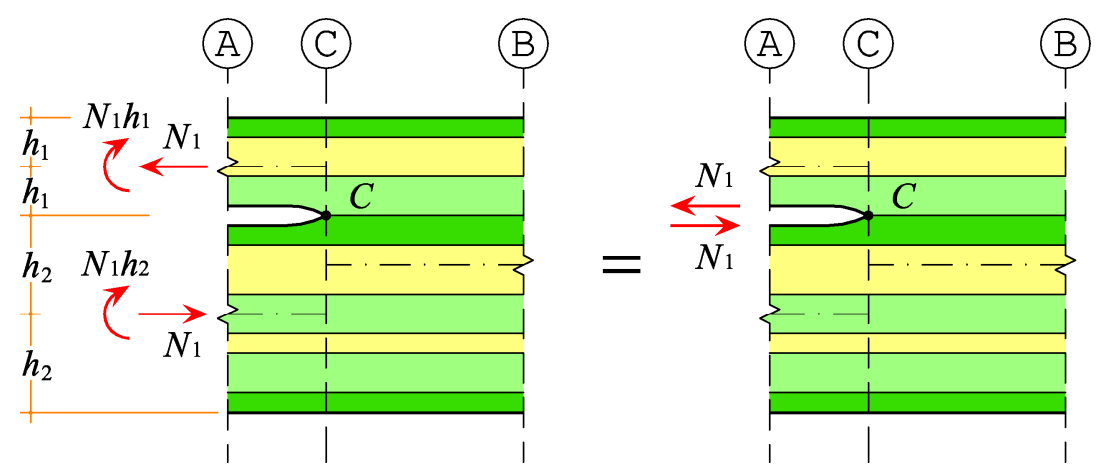

Fig. 22 Load condition analysed via the finite element method by Schapery and Davidson [17].

Schapery and Davidson [17] analysed a number of cases - including both homogeneous and bimaterial beams - and found that the values of $k_{1}$ are always close to zero. Hence, they concluded that further study would have been necessary to assess whether assuming $k_{1}=0$ could be a good approximation to evaluate the modal contributions without resorting to finite element calculations. In this respect, by comparing Eqs. (27) and (51) - regardless of the contribution of shear forces -, it can be seen that the present method is equivalent to Schapery and Davidson's method if the following expressions are assumed for their coefficients:

$k_{1}=0, \quad k_{2}=\sqrt{\frac{f_{u N} f_{\phi M}-f_{u M} f_{\phi N}}{f_{u N}}}, \quad k_{3}=\sqrt{f_{u N}}, \quad$ and $\quad k_{4}=\frac{f_{u M}}{\sqrt{f_{u N}}}$.

Interestingly, by substituting Eqs. (52) into the expressions in Appendix C, it is found that $N_{C}=N_{1}, \quad Q_{C}=0, \quad M_{C}=0$.

Then, by substituting Eqs. (54) into (27), the modal contributions are found as

$G_{\mathrm{I}}=0 \quad$ and $\quad G_{\mathrm{II}}=\frac{1}{2 B} f_{u N} N_{1}^{2}$ 
which means that, according to the present method, the problem of figure 22 is a case of pure mode II fracture.

\section{Discussion}

The predictions of the present method for the energy release rate and mode mixity have been compared to those of the elastic-interface model [35] and local method [11,22] for homogeneous and bimaterial delaminated beams.

For the axial force and bending moment load cases, the compared methods furnish the same results for the energy release rate. This is not surprising for the local method, which uses beam theory to compute $G$, except for the contribution of shear forces, which depends also on the root rotations $[22,23]$. For the elastic-interface model, this result can be explained by observing that for an infinitely long beam subjected to axial forces and bending moments, the elastic interface furnishes an additional term to the compliance, which does not depend on the delamination length, $a$. Therefore, this contribution vanishes when differentiating the compliance with respect to $a$ to obtain the energy release rate according to the well-known formula by Irwin and Kies [66]. Instead, the contribution to $G$ stemming from shear forces depends on the values of the elastic-interface constants, which are in turn related with the roots rotations (disregarded by the present approach). Anyway, when the (dimensionless) interface constants, $\mu_{x}$ and $\mu_{z}$, go to infinity, the predictions of the elastic-interface model converge to those of the present method.

As concerns the mode mixity, the predictions of the present method agree qualitatively with both the elastic-interface model and local method. However, differences in the values of the angle $\psi$ have been obtained. Such differences are most likely due to the simplifying assumptions on which the present method is based. The predictions of the elastic-interface model tend towards those of the present method as the interface constants, $\mu_{x}$ and $\mu_{z}$, increase and converge to the present method for $\mu_{x}=\mu_{z} \rightarrow+\infty$ (rigid interface limit case).

\section{Conclusions}

A method has been presented to calculate the energy release rate and mode mixity of laminated beams of rectangular cross section with through-the-width delaminations. According to classical lamination theory and Timoshenko beam kinematics, the axial, shear, and bending deformabilities, as well as the bending-extension coupling, have been taken into account. The kinematics of an elementary crack growth has been analysed by defining the 
crack-tip displacement rates as the relative displacements occurring at the crack tip per unit increase in crack length. Such newly defined quantities have been used to compute the cracktip forces. Furthermore, by adapting the virtual crack closure technique to the present context and assuming a suitable two-step process of closure of the crack, the energy release rate and its mode I and II contributions have been determined.

Results for homogeneous and bimaterial delaminated beams have been presented and compared to the predictions of the elastic-interface model [35] and local method [11, 22]. Perfect agreement between the compared methods has been obtained for the energy release rate due to axial forces and bending moments. Minor differences have been obtained for the shear force load case.

With respect to the mode mixity, qualitative agreement has been found between the compared methods, but some quantitative differences have emerged. Such differences are most likely due to the simplifying assumptions on which the present method is based. Taking into account some of the disregarded effects (root rotations, Poisson's effect, etc.) could probably help improve the accuracy of the present method. Furthermore, it has been observed that the results of the elastic-interface model converge to those of the present method for the limit case of a rigid interface, but is deduced in a completely independent way.

For general laminated beams, applications to some non-standard delamination toughness tests have been illustrated. Lastly, the present method has been compared to the method by Schapery and Davidson [17]. The two methods turn out to be equivalent if the coefficient $k_{1}$ defined in [17] is taken equal to zero. The present method nevertheless represents an improvement as it considers the effects of shear forces, not included in [17].

\section{Acknowledgements}

This manuscript was prepared during the author's one-semester sabbatical (Sept. 2014 Feb. 2015) at the Technical University of Denmark, DTU Wind Energy, Composites and Materials Mechanics Section. The author wishes to thank all the Section staff, in particular the Head of Section Professor Bent F. Sørensen, for hospitality, support, and scientific advice.

\section{Appendix A. Equivalent stiffnesses of a laminated beam}

The present study considers laminated beams of constant, rectangular cross section of width $B$ and height $H=2 h$ (Fig. A1). A reference system $O y z$ is fixed in the cross-section plane with the origin at the geometric centre of the section and the $y$-and $z$-axes aligned with the width and height directions, respectively. The beam is made of $n$ layers (or laminae) of 
different materials and thicknesses. Let $E_{x}^{(1)}, E_{x}^{(2)}, \ldots, E_{x}^{(n)}$ and $G_{z x}^{(1)}, G_{z x}^{(2)}, \ldots, G_{z x}^{(n)}$ respectively be the longitudinal Young's moduli and shear moduli of the $n$ layers. Furthermore, let $z_{0}, z_{1}, \ldots, z_{n}$ be the ordinates of the top and bottom surfaces of the layers.

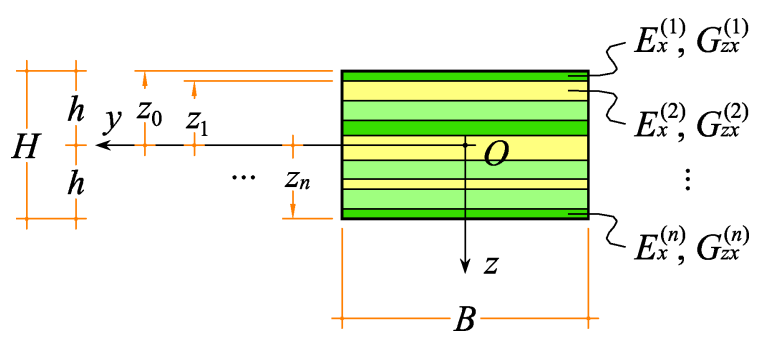

Fig. A1. Cross section of the laminated beam.

According to classical lamination theory [48], the laminated beam can be modelled as a homogeneous beam with the following equivalent stiffnesses:

$$
\begin{aligned}
& A=\sum_{i=1}^{n} E_{x}^{(i)}\left(z_{i}-z_{i-1}\right), \quad B=\frac{1}{2} \sum_{i=1}^{n} E_{x}^{(i)}\left(z_{i}^{2}-z_{i-1}^{2}\right), \\
& C=\frac{5}{6} \sum_{i=1}^{n} G_{z x}^{(i)}\left(z_{i}-z_{i-1}\right), \quad \text { and } \quad D=\frac{1}{3} \sum_{i=1}^{n} E_{x}^{(i)}\left(z_{i}^{3}-z_{i-1}^{3}\right) .
\end{aligned}
$$

In particular, $A, B, C$, and $D$, respectively denote the extensional stiffness, bendingextension coupling stiffness, shear stiffness, and bending stiffness per unit width of the laminated beam. Inversion of the constitutive laws for the beam leads to define the corresponding equivalent compliances,

$$
a=\frac{D}{A D-B^{2}}, \quad b=-\frac{B}{A D-B^{2}}, \quad c=\frac{1}{C}, \quad \text { and } \quad d=\frac{A}{A D-B^{2}} .
$$

\section{Appendix B. End-loaded laminated cantilever beam}

Let us consider a laminated cantilever beam of length $L$, loaded at its free end by an axial force, $\bar{N}$, a shear force, $\bar{Q}$, and a bending moment, $\bar{M}$ (Fig. B1). A reference system $O z x$ is fixed in the principal plane of the beam with the origin at the clamped end and the $x$-axis aligned with the longitudinal direction. Let $u$ and $w$ denote the axial and transverse displacements of the beam centreline, respectively, and $\phi$ the cross-section rotation, positive if counter-clockwise. Let $A, B, C$, and $D$ be the equivalent stiffnesses of the beam per unit width and let $a, b, c$, and $d$ be the corresponding compliances. 


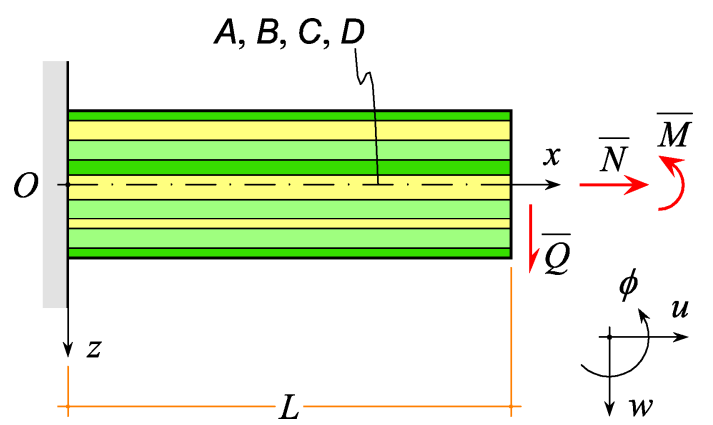

Fig. B1. Laminated cantilever beam loaded at its end.

The internal forces in the beam are

$N(x)=\bar{N}, \quad Q(x)=\bar{Q}, \quad$ and $\quad M(x)=\bar{M}-\bar{Q}(L-x)$.

Using the compatibility equations and inverse constitutive relationships for the laminated beam, the following relationships are obtained:

$\varepsilon=\frac{d u}{d x}=\frac{1}{B}(a N+b M), \quad \gamma=\frac{d w}{d x}+\phi=\frac{1}{B} c Q, \quad$ and $\quad \kappa=\frac{d \phi}{d x}=\frac{1}{B}(b N+d M)$,

where $B$ is the width of the cross section. By substituting Eqs. (B1) into (B2) and integrating with respect to $x$ (and also considering the boundary conditions at the clamped end, $x=0$ ), the generalised displacements of the beam centreline,

$$
\begin{aligned}
& u(x)=\frac{1}{B}\left[a \bar{N} x-b \bar{Q}\left(L-\frac{x}{2}\right) x+b \bar{M} x\right] \\
& w(x)=\frac{1}{B}\left\{-b \bar{N} \frac{x^{2}}{2}+\bar{Q}\left[c+d\left(L-\frac{x}{3}\right) \frac{x}{2}\right] x-d \bar{M} \frac{x^{2}}{2}\right\}, \quad \text { and } \\
& \phi(x)=\frac{1}{B}\left[b \bar{N} x-d \bar{Q}\left(L-\frac{x}{2}\right) x+d \bar{M} x\right]
\end{aligned}
$$

are obtained as functions of $x$. In particular, the generalised displacements at the loaded end, $x=L$, are

$$
\begin{aligned}
& \bar{u}=\frac{a L}{B} \bar{N}-\frac{b L^{2}}{2 B} \bar{Q}+\frac{b L}{B} \bar{M}, \\
& \bar{w}=-\frac{b L^{2}}{2 B} \bar{N}+\frac{1}{B}\left(c L+\frac{1}{3} d L^{3}\right) \bar{Q}-\frac{d L^{2}}{2 B} \bar{M}, \text { and } \\
& \bar{\phi}=\frac{b L}{B} \bar{N}-\frac{d L^{2}}{2 B} \bar{Q}+\frac{d L}{B} \bar{M} .
\end{aligned}
$$




\section{Appendix C. Crack-tip forces as functions of the internal forces}

By substituting Eqs. (9) into (16), after some calculations, the crack-tip forces,

$$
\begin{aligned}
& N_{C}=\frac{v_{1} N_{1}+v_{2} N_{2}+v_{3}\left(M_{1}+M_{2}\right)}{v_{4}}, \\
& Q_{C}=\frac{c_{1} Q_{1}-c_{2} Q_{2}}{C_{1}+c_{2}}, \text { and } \\
& M_{C}=\frac{\mu_{1} N_{1}+\mu_{2} N_{2}+\mu_{3} M_{1}+\mu_{4} M_{2}}{v_{4}},
\end{aligned}
$$

are obtained as functions of the internal forces in the sublaminates. In Eqs. (C1), the following constant parameters are used:

$$
\begin{aligned}
& v_{1}=a_{1}\left(d_{1}+d_{2}\right)-b_{1}\left(b_{1}+b_{2}\right)+b_{1} d_{2} h \\
& v_{2}=-a_{2}\left(d_{1}+d_{2}\right)+b_{2}\left(b_{1}+b_{2}\right)+b_{2} d_{1} h \\
& v_{3}=b_{1} d_{2}-b_{2} d_{1}+d_{1} d_{2} h \\
& v_{4}=\left(a_{1}+a_{2}\right)\left(d_{1}+d_{2}\right)-\left(b_{1}+b_{2}\right)^{2}+2\left(b_{1} d_{2}-b_{2} d_{1}\right) h+d_{1} d_{2} h^{2}
\end{aligned}
$$

and

$$
\begin{aligned}
& \mu_{1}=-a_{1} b_{2}+a_{2} b_{1}-a_{1}\left(d_{1} h_{1}-d_{2} h_{2}\right)+b_{1}^{2} h_{1}-b_{1} b_{2}\left(h+h_{2}\right)+b_{1} d_{2} h h_{2}, \\
& \mu_{2}=-a_{1} b_{2}+a_{2} b_{1}+a_{2}\left(d_{1} h_{1}-d_{2} h_{2}\right)+b_{2}^{2} h_{2}-b_{1} b_{2}\left(h+h_{1}\right)-b_{2} d_{1} h h_{1}, \\
& \mu_{3}=\left(a_{1}+a_{2}\right) d_{1}-b_{1}\left(b_{1}+b_{2}\right)+b_{1} d_{2} h_{2}-b_{2} d_{1}\left(h+h_{2}\right)+d_{1} d_{2} h h_{2}, \\
& \mu_{4}=-\left(a_{1}+a_{2}\right) d_{2}+b_{2}\left(b_{1}+b_{2}\right)+b_{2} d_{1} h_{1}-b_{1} d_{2}\left(h+h_{1}\right)-d_{1} d_{2} h h_{1} .
\end{aligned}
$$

The above expressions simplify significantly if the sublaminates are uncoupled in bendingextension $\left(b_{1}=b_{2}=0\right)$, in which case

$$
\begin{aligned}
& N_{C}=\frac{\left(d_{1}+d_{2}\right)\left(a_{1} N_{1}-a_{2} N_{2}\right)+d_{1} d_{2} h\left(M_{1}+M_{2}\right)}{\left(a_{1}+a_{2}\right)\left(d_{1}+d_{2}\right)+d_{1} d_{2} h^{2}} \\
& Q_{C}=\frac{c_{1} Q_{1}-c_{2} Q_{2}}{C_{1}+c_{2}}, \text { and } \\
& M_{C}=\frac{-\left(d_{1} h_{1}-d_{2} h_{2}\right)\left(a_{1} N_{1}-a_{2} N_{2}\right)+\left(a_{1}+a_{2}\right)\left(d_{1} M_{1}-d_{2} M_{2}\right)+d_{1} d_{2} h\left(h_{2} M_{1}-h_{1} M_{2}\right)}{\left(a_{1}+a_{2}\right)\left(d_{1}+d_{2}\right)+d_{1} d_{2} h^{2}} .
\end{aligned}
$$

\section{Appendix D. Elastic-interface model}

Liu et al. [35] have developed a general solution for the stress analysis of adhesively bonded joints. Their mechanical model consists of two homogeneous and isotropic, sheardeformable beams [47], partly connected by an elastic interface, in turn made of distributed normal and tangential springs. Their model can be used also for delaminated laminates, if the 
interface is interpreted as a conventional means to account for the laminate transverse deformability and not as representative of a physical layer of adhesive. The solution by Liu et al. [35] holds for beams of finite length. Here, the solution is presented for a semi-infinite bimaterial beam subjected to axial forces, shear forces, and bending moments (Fig. D1). This solution has been used for the comparisons shown in Section 4 of this paper.

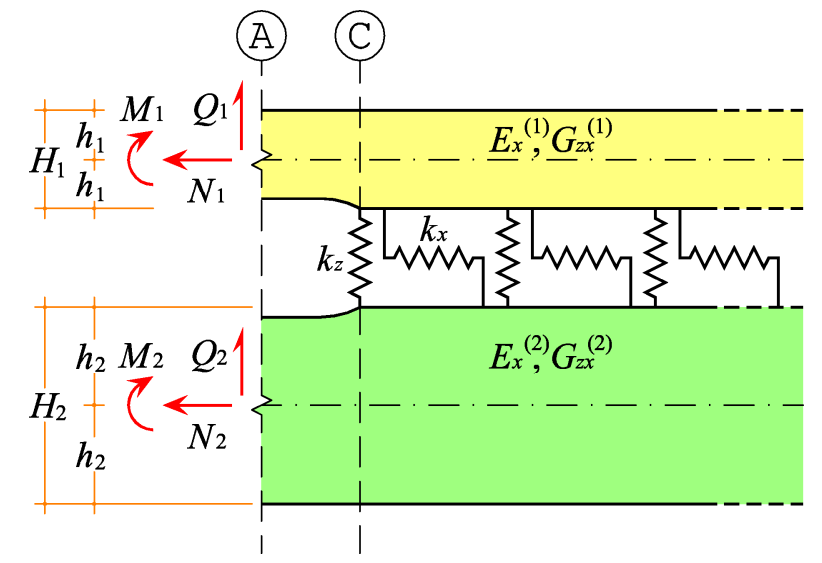

Fig. D1. Elastic-interface model of a semi-infinite bimaterial beam.

Let $E_{x}^{(1)}, G_{z x}^{(1)}$ and $E_{x}^{(2)}, G_{z x}^{(2)}$ respectively be the Young's modulus and shear modulus of the upper and lower beams. The equivalent stiffnesses can be computed from Eqs. (35) and the compliances from Eqs. (5). Let $k_{x}$ and $k_{z}$ respectively be the elastic constants of the distributed springs in the tangential and normal directions. The normal and tangential interfacial stresses attain peak values at the loaded cross section, whose values turn out to be $\sigma_{0}=F_{1}+F_{2}+F_{3}$ and $\tau_{0}=\frac{1}{b_{0}} \sum_{i=1}^{3}\left[\frac{1}{k_{z}} \frac{\Omega_{i}^{3 / 2}}{H^{3}}-\left(c_{1}+c_{2}\right) \frac{\Omega_{i}^{1 / 2}}{H}+\left(d_{1}+d_{2}\right) \frac{H}{\Omega_{i}^{1 / 2}}\right] F_{i}+F_{7}$,

where $b_{0}=d_{1} h_{1}-d_{2} h_{2}$ is the unbalance parameter (here, assumed $\left.b_{0} \neq 0\right), H=H_{1}+H_{2}$ is the total thickness, $F_{1}, F_{2}, F_{3}$, and $F_{7}$ are integration constants, and $\Omega_{1}, \Omega_{2}$, and $\Omega_{3}$ are the roots of the dimensionless characteristic equation,

$\Omega^{3}-\alpha_{1} \Omega^{2}+\alpha_{2} \Omega-\alpha_{3}=0$,

where

$$
\begin{aligned}
& \alpha_{1}=\left[k_{x}\left(a_{1}+a_{2}+d_{1} h_{1}^{2}+d_{2} h_{2}^{2}\right)+k_{z}\left(c_{1}+c_{2}\right)\right] H^{2}, \\
& \alpha_{2}=\left[k_{x} k_{z}\left(c_{1}+c_{2}\right)\left(a_{1}+a_{2}+d_{1} h_{1}^{2}+d_{2} h_{2}^{2}\right)+k_{z}\left(d_{1}+d_{2}\right)\right] H^{4}, \text { and } \\
& \alpha_{3}=k_{x} k_{z}\left[\left(a_{1}+a_{2}\right)\left(d_{1}+d_{2}\right)+d_{1} d_{2}\left(h_{1}+h_{2}\right)^{2}\right] H^{6} .
\end{aligned}
$$


The integration constants, $F_{1}, F_{2}, F_{3}$, and $F_{7}, F_{8}, F_{9}$, are calculated from the boundary conditions depending on the applied loads,

$$
\begin{aligned}
& N_{1}=\frac{B}{b_{0}} \sum_{i=1}^{3}\left[\frac{1}{k_{z}} \frac{\Omega_{i}}{H^{2}}-\left(c_{1}+c_{2}\right)+\left(d_{1}+d_{2}\right) \frac{H^{2}}{\Omega_{i}}\right] F_{i}-B F_{8} \text {, } \\
& Q_{1}=B \sum_{i=1}^{3} \frac{H}{\Omega_{i}^{1 / 2}} F_{i}+B\left[\frac{a_{1}+a_{2}}{d_{1}\left(h_{1}+h_{2}\right)}+h_{1}\right] F_{7} \text {, } \\
& M_{1}=B \sum_{i=1}^{3}\left\{\frac{h_{1}}{b_{0}}\left[\frac{1}{k_{z}} \frac{\Omega_{i}}{H^{2}}-\left(c_{1}+c_{2}\right)+\left(d_{1}+d_{2}\right) \frac{H^{2}}{\Omega_{i}}\right]-\frac{H^{2}}{\Omega_{i}}\right\} F_{i}+B \frac{a_{1} F_{8}+a_{2} F_{9}}{d_{1}\left(h_{1}+h_{2}\right)} \text {, } \\
& N_{2}=-\frac{B}{b_{0}} \sum_{i=1}^{3}\left[\frac{1}{k_{z}} \frac{\Omega_{i}}{H^{2}}-\left(c_{1}+c_{2}\right)+\left(d_{1}+d_{2}\right) \frac{H^{2}}{\Omega_{i}}\right] F_{i}+B F_{9} \text {, } \\
& Q_{2}=-B \sum_{i=1}^{3} \frac{H}{\Omega_{i}^{1 / 2}} F_{i}+B\left[\frac{a_{1}+a_{2}}{d_{2}\left(h_{1}+h_{2}\right)}+h_{2}\right] F_{7}, \quad \text { and } \\
& M_{2}=B \sum_{i=1}^{3}\left\{\frac{h_{2}}{b_{0}}\left[\frac{1}{k_{z}} \frac{\Omega_{i}}{H^{2}}-\left(c_{1}+c_{2}\right)+\left(d_{1}+d_{2}\right) \frac{H^{2}}{\Omega_{i}}\right]+\frac{H^{2}}{\Omega_{i}}\right\} F_{i}+B \frac{a_{1} F_{8}+a_{2} F_{9}}{d_{2}\left(h_{1}+h_{2}\right)} \text {. }
\end{aligned}
$$

From the peak values of the interfacial stresses, the modal contributions,

$G_{\mathrm{I}}=\frac{1}{2} \frac{\sigma_{0}^{2}}{k_{z}} \quad$ and $\quad G_{\mathrm{II}}=\frac{1}{2} \frac{\tau_{0}^{2}}{k_{x}}$

are determined. Lastly, the total energy release rate and mode-mixity angle are computed from Eqs. (21) and (29), respectively.

\section{References}

[1] Garg AC. Delamination - A damage mode in composite structures. Eng Fract Mech 1988;29:557-84.

[2] Sela N, Ishai O. Interlaminar fracture toughness and toughening of laminated composite materials: A review. Composites 1989;20:423-35.

[3] Bolotin VV. Delaminations in composite structures: Its origin, buckling, growth and stability. Composites Part B 1996;27:129-45.

[4] Tay TE. Characterization and analysis of delamination fracture in composites: An overview of developments from 1990 to 2001. Appl Mech Rev 2003;56:1-31.

[5] Senthil K, Arockiarajan A, Palaninathan R, Santhosh B, Usha KM. Defects in composite structures: Its effects and prediction methods - A comprehensive review. Compos Struct 2013;106:139-49.

[6] Hutchinson JW, Suo Z. Mixed mode cracking in layered materials. Adv Appl Mech 1991;29:63-191.

[7] Krueger R, Shivakumar K, Raju IS. Fracture mechanics analyses for interface crack problems. A review. In: 54th AIAA/ASME/ASCE/AHS/ASC Structures, Structural Dynamics, and Materials Conference; 8-11 Apr 2013; Boston, MA.

[8] Carlsson LA, Adams DF, Pipes RB. Experimental characterization of advanced composite materials. 4th 
ed. Boca Raton, FL: CRC Press; 2014.

[9] Ducept F, Gamby D, Davies P. A mixed-mode failure criterion derived from tests on symmetric and asymmetric specimens. Compos Sci Technol 1999;59:609-19.

[10] Williams JG. On the calculation of energy release rates for cracked laminates. Int J Fract 1988;36:101-19.

[11] Suo Z, Hutchinson JW. Interface crack between two elastic layers. Int J Fract 1990;43:1-18.

[12] Rybicki EF, Kanninen MF. A finite element calculation of stress intensity factors by a modified crack closure integral. Eng Fract Mech 1977;9:931-8.

[13] Krueger R. Virtual crack closure technique: History, approach, and applications. Appl Mech Rev 2004;57:109-43.

[14] Harvey CM, Wang S. Experimental assessment of mixed-mode partition theories. Compos Struct 2012;94:2057-67.

[15] Harvey CM, Eplett MR, Wang S. Experimental assessment of mixed-mode partition theories for generally laminated composite beams. Compos Struct 2015;124:10-8.

[16] Pook LP. Approximate stress intensity factors obtained from simple plate bending theory. Eng Fract Mech 1979;12:505-22.

[17] Schapery RA, Davidson BD. Prediction of energy release rate for mixed-mode delamination using classical plate theory. Appl Mech Rev 1990;43:S281-87.

[18] Nilsson KF, Storåkers B. On interface crack growth in composite plates. J Appl Mech 1992;59:530-38.

[19] Suo Z. Delamination specimens for orthotropic materials. J Appl Mech 1990;57:627-34.

[20] Suo Z, Bao G, Fan B, Wang TC. Orthotropy rescaling and implications for fracture in composites. Int J Solids Struct 1991;28:235-48.

[21] Bao G, Ho S, Suo Z, Fan B. The role of material orthotropy in fracture specimens for composites. Int J Solids Struct 1992;29:1105-16.

[22] Li S, Wang J, Thouless MD. The effects of shear on delamination of beam-like geometries. J Mech Phys Solids 2004;52:193-214.

[23] Andrews MG, Massabò R. The effects of shear and near tip deformations on energy release rate and mode mixity of edge-cracked orthotropic layers. Eng Fract Mech 2007;74:2700-20.

[24] Li W, Cheng G, Wang D, Wu J. A mixed mode partition method for delaminated beam structure. Eng Fract Mech 2015;148:15-26.

[25] Allix O, Ladeveze P. Interlaminar interface modelling for the prediction of delamination. Compos Struct $1992 ; 22: 235-42$.

[26] Corigliano D. Formulation, identification and use of interface models in the numerical analysis of composite delamination. Int J Solids Struct 1993;30:2779-811.

[27] Bruno D, Greco F. Mixed mode delamination in plates: a refined approach. Int J Solids Struct 2001;38:9149-77.

[28] Qiao P, Wang J. Mechanics and fracture of crack tip deformable bi-material interface. Int J Solids Struct 2004;41:7423-44.

[29] Szekrényes A, Uj J. Comparison of some improved solutions for mixed-mode composite delamination coupons. Compos Struct 2006;72:321-9.

[30] Bennati S, Valvo PS. Delamination growth in composite plates under compressive fatigue loads. Compos 
Sci Technol 2006;66:248-54.

[31] Bennati S, Colleluori M, Corigliano D, Valvo PS. An enhanced beam-theory model of the asymmetric double cantilever beam (ADCB) test for composite laminates. Compos Sci Techol 2009;69:1735-45.

[32] Bennati S, Fisicaro P, Valvo PS. An enhanced beam-theory model of the mixed-mode bending (MMB) test - Part I: Literature review and mechanical model. Meccanica 2013;48:443-62.

[33] Bennati S, Fisicaro P, Valvo PS. An enhanced beam-theory model of the mixed-mode bending (MMB) test - Part II: applications and results. Meccanica 2013;48:465-84.

[34] Cornetti P, Mantič V, Carpinteri A. Finite Fracture Mechanics at elastic interfaces. Int J Solids Struct 2012;49:1022-32.

[35] Liu Z, Huang Y, Yin Z, Bennati S, Valvo PS. A general solution for the two-dimensional stress analysis of balanced and unbalanced adhesively bonded joints. Int J Adhes Adhes 2014;54:112-23.

[36] de Borst R. Numerical aspects of cohesive-zone models. Eng Fract Mech 2003;70:1743-57.

[37] Yang Q, Cox B. Cohesive models for damage evolution in laminated composites. Int J Fract 2005;133:10737.

[38] Park K, Paulino GH. Cohesive zone models: A critical review of traction-separation relationships across fracture surfaces. Appl Mech Rev 2013;64:060802-1-20.

[39] Dimitri R, Trullo M, De Lorenzis L, Zavarise G. Coupled cohesive zone models for mixed-mode fracture: A comparative study. Eng Fract Mech 2015;148:145-79.

[40] Carlsson LA, Matteson RC, Aviles F, Loup DC. Crack path in foam cored DCB sandwich fracture specimens. Compos Sci Technol 2005;65:2612-21.

[41] Lundsgaard-Larsen C, Sørensen BF, Berggreen C, Østergaard RC. A modified DCB sandwich specimen for measuring mixed-mode cohesive laws. Eng Fract Mech 2008;75:2514-30.

[42] Rask M, Sørensen BF. Determination of the J integral for laminated double cantilever beam specimens: The curvature approach. Eng Fract Mech 2012;96:37-48.

[43] Wang S, Harvey CM. A theory of one-dimensional fracture. Compos Struct 2012;94:758-67.

[44] Harvey CM, Wang S. Mixed-mode partition theories for one-dimensional delamination in laminated composite beams. Eng Fract Mech 2012;96:737-59.

[45] Harvey CM, Wood JD, Wang S, Watson A. A novel method for the partition of mixed-mode fractures in 2D elastic laminated unidirectional composite beams. Compos Struct 2014;116:589-94.

[46] Williams JG. End corrections for orthotropic DCB specimens. Compos Sci Technol 1989;35:367-76.

[47] Timoshenko SP. Strength of materials: Elementary theory and problems. Vol. 1. Melbourne, FL: Krieger Publishing; 1984.

[48] Jones RM. Mechanics of composite materials. 2nd ed. Philadelphia, PA: Taylor \& Francis; 1999.

[49] Valvo PS. Fracture mode partition in delaminated beams using the crack-tip displacement rates. In: 18th European Conference on Fracture: Fracture of materials and structures from micro to macro scale; 30 Aug3 Sept 2010; Dresden, Germany.

[50] Valvo PS. A further step towards a physically consistent virtual crack closure technique. Int J Fract 2015;192:235-44.

[51] Valvo PS. A revised virtual crack closure technique for physically consistent fracture mode partitioning. Int J Fract 2012;173:1-20. 
[52] Irwin GR. Fracture. In: Flügge S, editor. Handbuch der Physik. Vol. VI, Berlin: Springer; 1958, p. 551-90.

[53] De Lorenzis L, Zavarise G. Modeling of mixed-mode debonding in the peel test applied to superficial reinforcements. Int J Solids Struct 2008;45:5419-36.

[54] Valvo PS. Does shear deformability influence the mode II delamination of laminated beams?. In: 17th European Conference on Fracture: Multilevel approach to fracture of materials, components and structures; 2-5 Sept 2008; Brno, Czech Republic.

[55] Mangalgiri PD, Johnson WS, Everett Jr RA. Effect of adherend thickness and mixed mode loading on debond growth in adhesively bonded composite joints. NASA Technical Memorandum 1986, NASA-TM88992.

[56] Sørensen BF, Jørgensen K, Jacobsen TK, Østergaard RC. DCB-specimen loaded with uneven bending moments. Int J Fract 2006;141:163-76.

[57] Reeder JR, Crews JH Jr. Mixed-mode bending method for delamination testing. AIAA J 1990;28:1270-6.

[58] ASTM D6671/D6671M-13. Standard test method for mixed Mode I-Mode II interlaminar fracture toughness of unidirectional fiber reinforced polymer matrix composites. ASTM International; 2013.

[59] Soboyejo WO, Lu GY, Chengalva S, Zhang J, Kenner V. A modified mixed-mode bending specimen for the interfacial fracture testing of dissimilar materials. Fatigue Fract Eng M 1999;22:799-810.

[60] Marannano GV, Pasta A. An analysis of interface delamination mechanisms in orthotropic and hybrid fiber-metal composite laminates. Eng Fract Mech 2007;74:612-26.

[61] Pereira AB, de Morais AB. Mixed mode I + II interlaminar fracture of carbon/epoxy laminates. Composites Part A 2008;39:322-33.

[62] Quispitupa A, Berggreen C, Carlsson LA. On the analysis of a mixed mode bending sandwich specimen for debond fracture characterization. Eng Fract Mech 2009;76:594-613.

[63] Shahverdi M, Vassilopoulos AP, Keller T. Mixed-mode I/II fracture behavior of asymmetric adhesivelybonded pultruded composite joints. Eng Fract Mech 2014;115:43-59.

[64] Ševčík M, Shahverdi M, Hutař P, Vassilopoulos AP. Analytical modeling of mixed-Mode bending behavior of asymmetric adhesively bonded pultruded GFRP joints. Eng Fract Mech 2015;147:228-42.

[65] Reeder JR, Demarco K, Whitley KS. The use of doubler reinforcement in delamination toughness testing. Composites Part A 2004;35:1337-44.

[66] Irwin GR, Kies JA. Critical energy release rate analysis of fracture strength. Weld J Res Suppl 1954;33:193-8. 